\title{
THE CENTRAL LIMIT THEOREM \\ FOR EMPIRICAL PROCESSES UNDER LOCAL CONDITIONS: THE CASE OF RADON INFINITELY DIVISIBLE LIMITS WITHOUT GAUSSIAN COMPONENT
}

\author{
NIELS T. ANDERSEN, EVARIST GINÉ AND JOEL ZINN
}

\begin{abstract}
Weak convergence results are obtained for empirical processes indexed by classes $\mathscr{F}$ of functions in the case of infinitely divisible purely Poisson (in particular, stable) Radon limits, under conditions on the local modulus of the processes $\{f(X): f \in \mathscr{F}\}$ ("bracketing" conditions). They extend (and slightly improve upon) a central limit theorem of Marcus and Pisier (1984) for Lipschitzian processes. The law of the iterated logarithm is also considered. The examples include Marcinkiewicz type laws of large numbers for weighted empirical processes and for the dual-bounded-Lipschitz distance between a probability in $\mathbf{R}$ and its associated empirical measures.
\end{abstract}

1. Introduction. Although there has been considerable activity regarding the CLT with Gaussian limits and $n^{1 / 2}$ normings for empirical processes indexed by classes of functions $\mathscr{F}$, the case of other stable limits and normings has not been considered, at least for large classes of functions $\mathscr{F}$. The object of this paper is to show that methods similar to those in Andersen et al. (1988) are appropriate to derive the CLT with $n^{1 / p}$ (or slightly more general) normings and $p$-stable Radon limits (or even, in the non-i.i.d. case, infinitely divisible Radon limits), under tail probability conditions for the local modulus of $\delta_{X}$ on $(\mathscr{F}, \rho)$, for appropriate distances $\rho$, that is, under bracketing. It is not surprising that such results can be obtained if one sees the bracketing CLT as an extension and improvement of the CLT for Lipschitz continuous processes (i.e., the Jain-Marcus CLT). Our previous results on bracketing may thus be viewed as the analogues of the Jain-Marcus (1974) CLT and the Ledoux (1981) LIL for processes with Lipschitz sample paths. Marcus and Pisier (1984) obtained CLT's with $n^{1 / p}$ norming and stable limits for such processes, and Ledoux (private communication) obtained recently another LIL; the results in this paper extend and slightly improve them. Actually, our framework for the CLT is more general and includes the non-i.i.d. case with convergence to other (necessarily infinitely divisible) Radon limits, as well as other normings in the stable case.

Received by the editors January 20, 1987.

1980 Mathematics Subject Classification (1985 Revision). Primary 60F17, 60F15, 60B12; Secondary $60 \mathrm{~F} 05,62 \mathrm{E} 20$.

Key words and phrases. Central limit theorems, law of the iterated logarithm, empirical processes, Marcinkiewicz laws of large numbers, bracketing conditions, majorizing measures.

Part of the research of the first author was carried out while visiting Texas A\&M University, supported by the Danish Natural Science Research Council.

The research of Giné and Zinn was partially supported by grants from the National Science Foundation, U.S.A. 
In $\S 2$ we give the asymptotic equicontinuity condition required to prove convergence of empirical processes to limits that are infinitely divisible measures without Gaussian component and that are Radon in $l^{\infty}(\mathscr{F})$. As it was to be expected, one needs the following two conditions for the CLT to hold: eventual tightness of the sums of the row laws outside neighborhoods of zero and convergence in probability to zero of the sums of the truncated variables, uniformly in $n$, as the truncation levels tend to zero (recall, in connection with this, Theorem 2.10 in Mandrekar and Zinn (1980)). Here, Hoffmann-Jørgensen's (1984) theory of weak convergence of sums of random elements taking values in nonseparable space is quite useful.

$\S 3$ contains a generalization of an inequality for sums of positive random variables that allowed replacing entropy by majorizing measure conditions in Andersen et al. (1988), and is used here for the same purpose.

In $\S 4$ we prove the main theorem, which is a CLT for the randomized empirical processes $\left\{\sum_{j=1}^{n} \varepsilon_{n j} f\left(X_{n j}\right) / b_{n}: f \in \mathscr{F}\right\}$ where $X_{n j}, j=1, \ldots, n$, are independent $(S, \mathscr{S})$-valued random variables and $\mathscr{F}$ is a class of measurable functions on $S$. The method of proof is similar to that of Theorem 3.1 in Andersen et al. (1988). Of course the majorizing measure and the local modulus conditions are quite different in the present situation.

In $\S 5$ we specialize the result from $\S 4$ to i.i.d. $X_{i}$ and $p$-stable limits, $p \in[1,2)$, with randomization replaced by centering at expectations for $p>1$. The norming constants $B(n)$ are a subset of all the possible ones in $\mathbf{R}$. Our methods do not seem to allow for all the normings $B(n)=n^{1 / p} L(n), L$ slowly varying, and the subset we can handle is related to the regularly varying functions used in Marcus (1987) for the study of infinitely divisible sample continuous processes whose Lévy measures split. For reasons of expediency, in the case $p=1$ only the norming $n$ and the randomized sums $\sum_{i=1}^{n} \varepsilon_{i} f\left(X_{i}\right)$ are considered. We also obtain results about stochastic boundedness and convergence to zero (in $\mathrm{pr}^{*}$ and a.s.) of the normalized sums $(n / B(n))\left(P_{n}-P\right)(f)$. In addition this section contains two instances of application of the general results, one to a slight extension and improvement of the CLT of Marcus and Pisier (1984) for Lipschitzian processes, and one to the CLT in $c_{0}$ showing that these results are sharp.

In $\S 6$ we apply a recent result of Ledoux and Talagrand that essentially reduces the law of the iterated logarithm in separable Banach spaces to a law of large numbers, to obtain a LIL under bracketing that complements the one obtained in Andersen et al. (1988) and Ledoux and Talagrand (1986). So, the unifying theme in this article is the use of majorizing measures combined with local conditions to prove convergence in probability to zero of randomized and suitably normalized empirical processes.

Finally, $\S 7$ contains Marcinkiewicz type laws of large numbers for weighted empirical processes and for the dual bounded Lipschitz norm between probability measures and their associated empiricals in the real line. Although our general theorems apply in these cases, they do not give the best results, which we obtain by other methods.

Notation and definitions are as in Andersen et al. (1988). When we refer to triangular arrays $X_{n j}$ of $S$-valued independent random variables and to independent Rademacher arrays $\left\{\varepsilon_{n j}\right\}$, we mean that these variables are defined on a product probability space, the $X_{n j}$ 's being coordinate functions and the $\left\{\varepsilon_{n j}\right\}$ being defined 
in another factor of the product, that is,

$$
(\Omega, \Sigma, \operatorname{Pr})=\left(\Pi_{n, j} S, \Pi_{n, j} \mathscr{S}, \Pi_{n j} P_{n j}\right) \times([0,1], \mathscr{B}, \lambda),
$$

the $X_{n j}$ are the coordinate functions $\Pi_{n, j} S \rightarrow S$ and the $\left\{\varepsilon_{n j}\right\}$ are independent Bernoulli symmetric random variables defined on $[0,1]$.

In a few instances we use the symbols $\gtrsim, \lesssim, \simeq$ to denote inequalities that hold up to constant multipliers $c \in\left(10^{-6}, 10^{6}\right)$.

We recall the following two exponential inequalities, to be used in what follows: (a) Bernstein's inequality (e.g., Bennett (1962)): if $\xi_{i}$ are independent centered real random variables with ess $\sup \left|\xi_{i}\right| \leq c<\infty, i \leq n$, then

$$
P\left\{\sum_{i=1}^{n} \xi_{i}>t\right\} \leq \exp \left\{-t^{2} /\left(2 \sum_{i=1}^{n} E \xi_{i}^{2}+\frac{2 t c}{3}\right)\right\}, \quad t>0 .
$$

(b) Upper bound for binomial probabilities (Giné and Zinn (1984)): if $A_{j}$ are independent events with $p_{j}=P A_{j}$, then

$$
P\left\{\sum_{i=1}^{n} I_{A_{j}} \geq l\right\} \leq\left(\frac{e \sum_{j=1}^{n} p_{j}}{l}\right)^{l}, \quad 1 \leq l \leq n, n \in \mathbf{N} .
$$

2. The CLT with non-Gaussian limits in $l^{\infty}(\mathscr{F})$. In this section we show how the CLT in the non-Gaussian case reduces to conditions on the individual summands together with probability inequalities. Stochastic boundedness is also considered.

Let $\mathscr{F}$ be an index set and let $l^{\infty}(\mathscr{F})$ be the Banach space of bounded real functions on $\mathscr{F}$ with the sup norm $\|\cdot\| \mathscr{F}$. A random element $\mathbf{X}$ in $l^{\infty}(\mathscr{F})$ is a mapping $\mathbf{X}:(\Omega, \Sigma, \operatorname{Pr}) \rightarrow l^{\infty}(\mathscr{F})$ such that $\lim _{M \rightarrow \infty} \operatorname{Pr}^{*}\left\{\|\mathbf{X}\|_{\mathscr{F}}>M\right\}=0$. The following definition is taken from Hoffmann-Jørgensen (1984).

2.1. DEFINITION. (1) $\left\{\mathbf{X}_{n}\right\}_{n=1}^{\infty}$ is an eventually tight sequence if for every $\varepsilon>0$ there is a compact set $K \in l^{\infty}(\mathscr{F})$ such that

$$
\limsup _{n \rightarrow \infty} \operatorname{Pr}^{*}\left\{\mathbf{X}_{n} \in G^{c}\right\}<\varepsilon
$$

for all open sets $G \supset K$. Since $l^{\infty}(\mathscr{F})$ is a Banach space $K$ and $G$ may be taken to be convex and symmetric in this definition.

(2) $\left\{\mathbf{X}_{n}\right\}_{n=1}^{\infty}$ converges in law to a Radon limit if there exists a Radon probability measure $\gamma$ on $l^{\infty}(\mathscr{F})$ such that for all $H: l^{\infty}(\mathscr{F}) \rightarrow \mathbf{R}$ bounded and continuous,

$$
\lim _{n \rightarrow \infty} E^{*} H\left(\mathbf{X}_{n}\right)=\int H d \gamma
$$

(3) $\left\{\mathbf{X}_{n}\right\}_{n=1}^{\infty}$ is stochastically bounded if

$$
\lim _{M \rightarrow \infty} \limsup _{n} \operatorname{Pr}^{*}\left\{\left\|\mathbf{X}_{n}\right\|_{\mathscr{F}}>M\right\}=0 .
$$

Given a random element $\mathbf{X}$ in $l^{\infty}(\mathscr{F})$ define a set function $\mathscr{L}^{*}(X)$ on the subsets of $l^{\infty}(\mathscr{F})$ by

$$
\mathscr{L}^{*}(\mathbf{X})(A)=\operatorname{Pr}^{*}\{\mathbf{X} \in A\}, \quad A \in l^{\infty}(\mathscr{F}),
$$

and for any $B \subset l^{\infty}(\mathscr{F})$,

$$
\left.\mathscr{L}^{*}(\mathbf{X})\right|_{B}(A)=\operatorname{Pr}^{*}\{\mathbf{X} \in A \cap B\}, \quad A \subset l^{\infty}(\mathscr{F}) .
$$


2.1'. Definition. Given $\left\{\mathbf{X}_{n j}: j \leq n, n \in \mathbf{N}\right\}$, we say that the sequence of set functions $\left\{\left.\sum_{j} \mathscr{L}^{*}\left(\mathbf{X}_{n j}\right)\right|_{\left[\|x\|_{\mathscr{g}}>\delta\right]}\right\}_{n=1}^{\infty}$ is eventually tight if both

$$
\sup _{n} \sum_{j} \operatorname{Pr}^{*}\left\{\left\|\mathbf{X}_{n j}\right\|_{\mathscr{F}}>\delta\right\}<\infty
$$

and for every $\varepsilon>0$ there exists $K$ compact (convex, symmetric) such that for all open (convex, symmetric) sets $G \supset K$,

$$
\limsup _{n \rightarrow \infty} \sum_{j} \operatorname{Pr}^{*}\left\{\mathbf{X}_{n j} \in G^{c},\left\|\mathbf{X}_{n j}\right\|_{\mathscr{F}}>\delta\right\}<\varepsilon
$$

The following statement is Mandrekar and Zinn's (1980, Theorem 2.10) restated in the nonseparable, nonmeasurable case. The proof is somewhat different.

2.2. Proposition. Let $\left\{\mathbf{X}_{n j}: j=1, \ldots, n ; n \in \mathbf{N}\right\}$ be a triangular array of $l^{\infty}(\mathscr{F})$-valued random elements. Assume:

(i) The sequence of set functions

$$
\left\{\left.\sum_{j=1}^{n} \mathscr{L}^{*}\left(\mathbf{X}_{n j}\right)\right|_{\left[\|x\|_{. \rightarrow}>\delta\right]}\right\}_{n=1}^{\infty}
$$

is eventually tight for every $\delta>0$, and

(ii) for all $\varepsilon>0$,

$$
\lim _{\delta \rightarrow 0} \limsup _{n \rightarrow \infty} \operatorname{Pr}^{*}\left\{\left\|\sum_{j=1}^{n} \mathbf{X}_{n j} I_{\left[\left\|\mathbf{X}_{n j}\right\|, \Omega \delta\right]}\right\|_{\mathscr{F}}>\varepsilon\right\}=0 .
$$

Then the sequence of random elements $\left\{\sum_{j=1}^{n} \mathbf{X}_{n j}\right\}_{n=1}^{\infty}$ is eventually tight.

PROOF. We first show that for all $\delta>0\left\{\sum_{j=1}^{n} \mathbf{X}_{n j} I_{\left[\left\|\mathbf{X}_{n j}\right\|, g^{\prime}>\delta\right]}\right\}_{n=1}^{\infty}$ is an eventually tight sequence. For this we follow the proof of Lemma 2.4 in de Acosta, Araujo and Giné (1978). We let $\mathbf{X}_{n j}^{\delta}=\mathbf{X}_{n j} I_{\left[\left\|\mathbf{X}_{n j}\right\|_{\mathscr{s}}>\delta\right]}$. For $G$ convex, symmetric, open, $q_{G}$ denotes its Minkowski functional. Let $\tilde{K}$ be a compact, convex symmetric set such that

$$
\limsup _{n \rightarrow \infty} \sum_{j=1}^{n} \operatorname{Pr}^{*}\left\{q_{\tilde{G}}\left(\mathbf{X}_{n j}^{\delta}\right)>1\right\} \leq \varepsilon / 2
$$

for all $\tilde{G} \supset \tilde{K}$ open, convex, symmetric. Let $m$ be such that

$$
\sum_{j=1}^{n} \operatorname{Pr}^{*}\left\{\left\|\mathbf{X}_{n j}\right\|_{\mathscr{F}}>\delta\right\} \leq \varepsilon m / 2
$$

$m$ and $\tilde{K}$ exist by hypothesis. Define $K=m \tilde{K}$ and $G=m \tilde{G}$; then, as $\tilde{G}$ runs over all convex symmetric open neighborhoods of $\tilde{K}, G$ runs over all such neighborhoods of $K$. Since

$$
q_{G}\left(\sum_{n=1}^{n} \mathbf{X}_{n j}^{\delta}\right) \leq \sum_{j=1}^{n} q_{G}\left(\mathbf{X}_{n j}^{\delta}\right) \leq\left(\sum_{j=1}^{n} I_{\left[\left\|\mathbf{X}_{n j}\right\|_{\mathcal{F}}>\delta\right]}\right) \max _{j \leq n} q_{G}\left(\mathbf{X}_{n j}^{\delta}\right),
$$


we have

$$
\begin{aligned}
\operatorname{Pr}^{*}\left\{q_{G}\left(\sum_{j=1}^{n} \mathbf{X}_{n j}^{\delta}\right)>1\right\} \leq m^{-1} E^{*} \sum_{j=1}^{n} I_{\left[\left\|\mathbf{X}_{n j}\right\|_{\mathscr{G}}>\delta\right]} \\
\quad+\operatorname{Pr}^{*}\left\{q_{G}\left(\sum_{j=1}^{n} \mathbf{X}_{n j}^{\delta}\right)>1, \sum_{j=1}^{n} I_{\left.\left[\left\|\mathbf{X}_{n j}\right\|_{\mathscr{g}}>\delta\right] \leq m\right\}} \leq\right. \\
\leq m^{-1} \sum_{j=1}^{n} \operatorname{Pr}^{*}\left\{\left\|\mathbf{X}_{n j}\right\|_{\mathscr{g}}>\delta\right\}+\operatorname{Pr}^{*}\left\{\max _{j \leq n} q_{G}\left(\mathbf{X}_{n j}^{\delta}\right)>1 / m\right\} \\
\leq m^{-1} \sum_{j=1}^{n} \operatorname{Pr}^{*}\left\{\left\|\mathbf{X}_{n j}\right\|_{\mathscr{G}}>\delta\right\}+\sum_{j=1}^{n} \operatorname{Pr}^{*}\left\{q_{\tilde{G}}\left(\mathbf{X}_{n j}^{\delta}\right)>1\right\} .
\end{aligned}
$$

Hence by (2.5) and (2.6), $\limsup _{n \rightarrow \infty} \operatorname{Pr}^{*}\left\{q_{G}\left(\sum_{j=1}^{n} \mathbf{X}_{n j}^{\delta}\right)>1\right\}<\varepsilon$. This proves that $\left\{\sum_{j=1}^{n} \mathbf{X}_{n j}^{\delta}\right\}_{n=1}^{\infty}$ is eventually tight.

Now we apply this fact together with hypothesis (2.4) to prove the proposition. We use Example 7.28 in Hoffmann-Jørgensen (1984) (see also Andersen and Dobrić (1987, Theorem 2.12)). Set $\mathbf{X}_{n j} I_{\left[\left\|\mathbf{X}_{n j}\right\|_{\mathcal{F}}<\delta\right]}=\mathbf{X}_{n j \delta}$. Given a pseudo-metric $\rho$ on $\mathscr{F}$, let $w_{\rho}(x, \varepsilon)=\sup _{\rho(f, g) \leq \varepsilon}|x(f)-x(g)|, x \in l^{\infty}(\mathscr{F}), \varepsilon>0$, be its oscillation function. We have, for $\delta>0, \varepsilon>0$,

$$
\begin{aligned}
\operatorname{Pr}^{*}\left\{w_{\rho}\left(\sum_{j=1}^{n} \mathbf{X}_{n j}, \varepsilon\right)>\varepsilon\right\} \\
\leq \operatorname{Pr}^{*}\left\{\left\|\sum_{j=1}^{n} \mathbf{X}_{n j \delta}\right\|_{\mathscr{F}}>\varepsilon / 4\right\}+\operatorname{Pr}^{*}\left\{w_{\rho}\left(\sum_{j=1}^{n} \mathbf{X}_{n j}^{\delta}, \varepsilon\right)>\varepsilon / 2\right\} .
\end{aligned}
$$

Given $\varepsilon>0$, choose $\delta>0$ such that

$$
\underset{n}{\lim \sup \operatorname{Pr}^{*}}\left\{\left\|\sum_{j=1}^{n} \mathbf{X}_{n j \delta}\right\|_{\mathscr{F}}>\varepsilon / 4\right\}<\varepsilon / 2,
$$

which is possible by (2.4). Also, by Example 7.28 in Hoffmann-Jørgensen (1984) and eventual tightness of $\left(\sum_{j=1}^{n} \mathbf{X}_{n j}^{\delta}\right\}_{n=1}^{\infty}$ there exists a totally bounded pseudo-metric $\rho$ on $\mathscr{F}$ such that

$$
\underset{n}{\lim \sup } \operatorname{Pr}^{*}\left\{w_{\rho}\left(\sum_{j=1}^{n} \mathbf{X}_{n j}^{\delta}, \varepsilon\right)>\varepsilon / 2\right\} \leq \varepsilon / 2 .
$$

For such $\rho$ we thus have

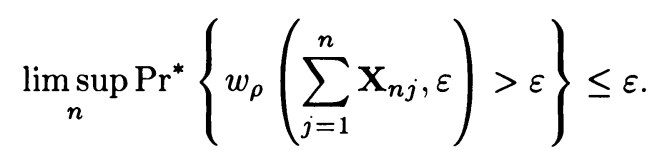


A standard argument using the same facts about $\mathbf{X}_{n j \delta}$ and $\mathbf{X}_{n j}^{\delta}$ shows that $\left\{\sum_{j=1}^{n} \mathbf{X}_{n j}\right\}_{n=1}^{\infty}$ is stochastically bounded; that is,

$$
\lim _{M \rightarrow \infty} \limsup _{n \rightarrow \infty} \operatorname{Pr}^{*}\left\{\left\|\sum_{j=1}^{n} \mathbf{X}_{n j}\right\|_{\mathscr{F}}>M\right\}=0 .
$$

But this and (2.7) imply eventual tightness of this sequence, again by Example 7.28 in Hoffmann-Jørgensen, loc. cit.

We say that the finite dimensional distributions (f.d.d.) of $\left\{\mathbf{X}_{n}\right\}_{n=1}^{\infty}$ converge, if for each $k \in \mathbf{R}$ and $f_{1}, \ldots, f_{k} \in \mathscr{F}$, the sequence $\left(\mathbf{X}_{n}\left(f_{1}\right), \ldots, \mathbf{X}_{n}\left(f_{k}\right)\right) \in \mathbf{R}^{k}$ converges in law. The previous proposition together with Theorem $7.11\left(3^{\circ}\right)$ of Hoffmann-Jørgensen (1984) (applied with

$$
\psi=\left\{\exp \left(i \sum_{\text {finite }} \alpha_{k} f_{k}\right): \alpha_{k} \in \mathbf{R}, f_{k} \in \mathscr{F}\right\} \subset C\left(l^{\infty}(\mathscr{F})\right),
$$

where $\sum \alpha_{k} f_{k}(x):=\sum \alpha_{k} x\left(f_{k}\right)$ for $\left.x \in l^{\infty}(\mathscr{F})\right)$ gives

2.3. COROLlary. If conditions (i) and (ii) of Proposition 2.2 hold, and if moreover the f.d.d. of $\left\{\sum_{j=1}^{n} \mathbf{X}_{n j}\right\}_{n=1}^{\infty}$ converge (in law) then this sequence converges in law to a Radon limit in $l^{\infty}(\mathscr{F})$.

2.4. REMARK. A proof similar (but easier) to that of Proposition 2.2 shows the following: Let $\left\{\mathbf{X}_{n j}: j=1, \ldots, n ; n \in \mathbf{N}\right\}$ be a triangular array of $l^{\infty}(\mathscr{F})$-valued random elements. Assume

$$
\lim _{M \rightarrow \infty} \limsup _{n \rightarrow \infty} \sum_{j=1}^{n} \operatorname{Pr}^{*}\left\{\left\|\mathbf{X}_{n j}\right\|_{\mathscr{F}}>M\right\}=0
$$

with $\sup _{n} \sum_{j=1}^{n} \operatorname{Pr}^{*}\left\{\left\|\mathbf{X}_{n j}\right\|_{\mathscr{F}}>M\right\}<\infty$ for all $M>\delta_{0}$, for some $\delta_{0}>0$, and

$$
\lim _{M \rightarrow \infty} \limsup _{n \rightarrow \infty} \operatorname{Pr}^{*}\left\{\left\|\sum_{j=1}^{n} \mathbf{X}_{n j} I_{\left[\left\|\mathbf{X}_{n j}\right\|_{.} \leq \delta\right]}\right\|_{\mathscr{F}}>M\right\}=0
$$

for some $\delta>\delta_{0}$. Then the sequence $\left\{\sum_{j=1}^{n} \mathbf{X}_{n j}\right\}_{n=1}^{\infty}$ is stochastically bounded.

For applications to empirical processes, the index set $\mathscr{F}$ is a subset of $\mathscr{L}_{0}(S, \mathscr{S})$ with envelope $F(s):=\sup _{f \in \mathscr{F}}|f(s)|$ finite for all $s \in S$, and $\mathbf{X}_{n j}=\delta_{X_{n j}} / b_{n}$ or $\mathbf{X}_{n j}=\varepsilon_{n j} \delta_{X_{n j}} / b_{n}$, where $\left\{X_{n j}\right\}$ is a triangular array of $(S, \mathscr{S})$-valued random variables, the $b_{n}$ 's are positive real constants, and $\left\{\varepsilon_{n j}\right\}$ is a Rademacher array independent of $\left\{X_{n j}\right\}$ (see the last paragraph of $\S 1$ for the precise set-up). Moreover the $X_{n j}$ 's are row-wise independent (i.e., for each $n$, the random variables $X_{n 1}, \ldots, X_{n n}$ are independent; in fact, they are coordinates of a large product probability space). Actually our main interest is in the case $\mathbf{X}_{n j}=\delta_{X_{j}} / b_{n}$ with $X_{j}$ i.i.d. and $b_{n} \uparrow \infty$, that is, the $p$-stable limit case, in fact only for $p \in[1,2)$ : the case $p=2$ is considered in Andersen et al. (1988) and the case $p<1$ is trivial in the sense that condition (ii) of Proposition 2.2 becomes superfluous (as $\left.b_{n} / n \rightarrow \infty\right)$ and condition (i) is also necessary for the CLT, at least in the measurable case. Another reason for not considering the case $p<1$ is that it has no relevance regarding the law of large numbers. 
In the $p$-stable case with $p \neq 1$ we are interested in the convergence in law of $\left\{\sum_{j=1}^{n}\left(f\left(X_{j}\right)-P f\right) / b_{n}: f \in \mathscr{F}\right\}_{n=1}^{\infty}$. The following is a convenient complement to Proposition 2.2 in this case.

2.5. Proposition. Let $\left\{X_{i}\right\}_{i=1}^{\infty}$ be i.i.d. $(S, \mathscr{S})$-valued random variables with law $P$ and let $\left\{\varepsilon_{i}\right\}_{i=1}^{\infty}$ be a Rademacher sequence independent of $\left\{X_{i}\right\}_{i=1}^{\infty}$. Let $\mathscr{F} \subset \mathscr{L}_{1}(S, \mathscr{S}, P)$ have everywhere finite envelope $F$. Assume there exist $b_{n} \uparrow \infty$ such that:

(i) the f.d.d. of the sequence of random elements of $l^{\infty}(\mathscr{F})$

$$
\left\{\sum_{j=1}^{n}\left(f\left(X_{j}\right)-P f\right) / b_{n}: f \in \mathscr{F}\right\}_{n=1}^{\infty}
$$

converge in law to p-stable probability measures in Euclidean space, for a fixed $p \in(1,2)$.

(ii) The sequence of set functions of $l^{\infty}(\mathscr{F})$,

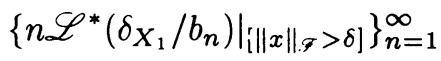

is eventually tight for all $\delta>0$.

(iii) For all $\varepsilon>0$,

$$
\lim _{\delta \rightarrow 0} \limsup _{n} \operatorname{Pr}^{*}\left\{\left\|\sum_{j=1}^{n} \varepsilon_{j} f I_{\left[F \leq \delta b_{n}\right]}\left(X_{j}\right) / b_{n}\right\|_{\mathscr{F}}>\varepsilon\right\}=0 .
$$

Then the sequence (2.8) converges in law to a p-stable Radon probability measure in $l^{\infty}(\mathscr{F})$.

2.6. Notation. If the conclusion of Proposition 2.5 holds for $\mathscr{F}, P$, and $\left\{b_{n}\right\}$,we write $\mathscr{F} \in \operatorname{CLT}\left(P ; b_{n}\right)$.

PROOF OF PROPOSITION 2.5 (SKETCH). Convergence of the f.d.d. of $\left\{\sum_{j=1}^{n}\left(f\left(X_{j}\right)-P\right) / b_{n}\right\}$ implies the same for $\left\{\sum_{j=1}^{n} \varepsilon_{j} f\left(X_{j}\right) / b_{n}\right\}$. Condition (ii) also holds for $\varepsilon \delta_{X_{1}}$. So, Corollary 2.3 gives convergence in law to a Radon measure of the randomized sequence $\left\{\sum_{j=1}^{n} \varepsilon_{j} f\left(X_{j}\right) / b_{n}: f \in \mathscr{F}\right\}$. The limit is $p$-stable and, in analogy to the Gaussian case, $p$-stable Radon measures satisfy $\gamma_{*}\left(C_{u}(\mathscr{F}, \rho)\right)=1$ where $\rho(f, g)=\Lambda_{p, \infty}^{\gamma}(|f-g|) \simeq \int|f-g| d \gamma$, and $(\mathscr{F}, \rho)$ is totally bounded (see Andersen and Dobric (1987) for the first statement and Marcus and Pisier (1985) for the second). So, the randomized sequence is eventually uniformly $\rho$-equicontinuous (as e.g. in the proof of Theorem 1.1.3 in Giné and Zinn (1986)). This implies, as e.g. in Giné and Zinn (1984, proof of Corollary 2.13), that

$$
\lim _{\delta \rightarrow 0} \limsup _{n} n \operatorname{Pr}^{*}\left\{\sup _{f, g \in \mathscr{F}, \rho(f, g) \leq \delta}|f-g|\left(X_{1}\right) / b_{n}>\varepsilon\right\}=0 .
$$

Then using regular variation of $\left\{b_{n}\right\}$ and the theorem in Feller $(1971$, p. 281) as in the proof of the stable CLT in $R$, it follows that

$$
\lim _{\delta \rightarrow 0} \limsup _{n} \sup _{f, g \in \mathscr{F}, \rho(f, g) \leq \delta} \operatorname{Pr}\left\{\left|\sum_{j=1}^{n}\left((f-g)\left(X_{i}\right)-P(f-g)\right)\right| / b_{n}>\varepsilon\right\}=0 .
$$


This allows use of the randomization lemmas in $§ 2$ of Giné and Zinn (1984) to obtain the eventual uniform $\rho$-equicontinuity of the nonrandomized sequence (2.8), hence its eventual tightness and, by (i), its convergence in law to a Radon limit.

2.7. REMARK. Regarding stochastic boundedness in the i.i.d. case Remark 2.4 gives the following statement: Let $\left\{X_{i}\right\}_{i=1}^{\infty}$ be i.i.d. $(S, \mathscr{S})$-valued random variables with law $P$ and let $\left\{\varepsilon_{i}\right\}_{i=1}^{\infty}$ be a Rademacher sequence independent of $\left\{X_{i}\right\}_{i=1}^{\infty}$. Let $\mathscr{F} \subset \mathscr{L}_{1}(S, \mathscr{S}, P)$ with envelope $F$ everywhere finite. Assume there exists a strictly increasing sequence $\left\{b_{n}\right\}$ regularly varying of exponent $1 / p$ for some $p \in(1,2)$ such that

(i) $\sup _{n} n P^{*}\left\{F>b_{n}\right\}<\infty$ and

(ii) for some $\delta>0$,

$$
\lim _{M \rightarrow \infty} \limsup _{n \rightarrow \infty} \operatorname{Pr}^{*}\left\{\left\|\sum_{j=1}^{n} \varepsilon_{j} f I_{\left[F \leq \delta b_{n}\right]}\left(X_{j}\right)\right\|_{\mathscr{F}} / b_{n}>M\right\}=0 .
$$

Then the sequence $\left\{\left\|\sum_{j=1}^{n}\left(f\left(X_{i}\right)-P f\right) / b_{n}\right\|_{\mathscr{F}}\right\}_{n=1}^{\infty}$ is stochastically bounded. (In this case desymmetrization again follows from the lemmas in Giné and Zinn $(1984, \S 2)$, since condition (i) implies

$$
\lim _{M \rightarrow \infty} \limsup _{n \rightarrow \infty} \sup _{f \in \mathscr{F}} \operatorname{Pr}\left\{\left|\sum_{j=1}^{n}\left(f\left(X_{j}\right)-P f\right) / b_{n}\right|>M\right\}=0
$$

by the usual computations with regularly varying functions, as mentioned in the proof of Proposition 2.5.)

3. An exponential bound for sums of positive, bounded random variables. One of the basic technical tools in proofs of limit theorems combining bracketing and majorizing measures is the inequality in this section. The present inequality generalizes the one in Andersen et al. (1988).

3.1. THEOREM. Let $\left\{\xi_{i}: i \leq n\right\}$ be independent, nonnegative real random variables and let $\psi: \mathbf{R}^{+} \rightarrow \mathbf{R}^{+}$be a strictly increasing function. Assume there exist $h_{l} \downarrow 0$ and $r \geq 1$ such that

(i) $\sum_{i=1}^{n} P\left\{\xi_{i}>h_{l}\right\} \leq l / 3, l \geq r$,

(ii) $h_{l} \leq \psi\left(1 / \sum_{i=1}^{l} h_{i}\right), l \geq r$.

Then, for $d \geq 1, c>4 d$ and $b>0$ satisfying

(iii) $\psi(b) \leq h_{r}$

(iv) $4 r b \psi(b) \leq c$,

the following inequality holds:

$$
\begin{aligned}
P\left\{\sup _{a>0} a \sum_{i=1}^{n} \xi_{i} I_{\left[\psi(a)<\xi_{i} \leq d \psi(b)\right]}>c\right\} \\
\leq 12 \exp \{-c \ln (3 / e) / 2(d \vee 2) b \psi(b)\} .
\end{aligned}
$$


ProOF. We have

$$
\begin{aligned}
& P\left\{\sup _{0<a \leq b} a \sum_{i=1}^{n} \xi_{i} I_{\left[\psi(a)<\xi_{i} \leq d \psi(b)\right]}>c\right\} \\
& \leq P\left\{\sup _{0<a \leq b} a \sum_{i=1}^{n} \xi_{i} I_{\left[\psi(a)<\xi_{i} \leq \psi(b)\right]}>c / 2\right\} \\
&+P\left\{\sum_{i=1}^{n} I_{\left[\psi(b)<\xi_{i} \leq d \psi(b)\right]}>c / 2 b d \psi(b)\right\}:=(\mathrm{I})+(\mathrm{II}) .
\end{aligned}
$$

Since $h_{l} \downarrow 0$ we can find $l_{0} \geq r$ such that $h_{l_{0}+1} \leq \psi(b) \leq h_{l_{0}}$ by (iii). Then, using (ii) and the fact that $\psi$ is increasing, we have $b \leq 1 / \sum_{i=1}^{l_{0}} h_{i} \leq 1 / l_{0} h_{l_{0}}$, i.e. $l_{0} \leq 1 / b h_{l_{0}} \leq 1 / b \psi(b)$. So, by inequalities (1.2) and (i) we obtain the following estimate for (II):

$$
\begin{aligned}
(\mathrm{II}) & \leq\left[2 c^{-1} d e b \psi(b) \sum_{i=1}^{n} P\left\{\xi_{i} \geq \psi(b)\right\}\right]^{c / 2 d b \psi(b)} \\
& \leq\left(2 c^{-1} d e b \psi(b)\left(l_{0}+1\right) / 3\right)^{c / 2 d b \psi(b)} \\
& \leq(4 d e / 3 c)^{c / 2 d b \psi(b)} \leq \exp \left\{-\frac{c \ln (3 / e)}{2 d b \psi(b)}\right\}
\end{aligned}
$$

since $c>4 d$.

Now we estimate (I). Let $\zeta_{i}=\xi_{i} I_{\left[\xi_{i} \leq \psi(b)\right]}$ and let $\zeta_{i}^{*}, 1 \leq i \leq n$, be the nonincreasing order statistics of $\zeta_{i}, 1 \leq i \leq n, \varsigma_{n+1}^{*}:=0$. Put $\lambda=c / 4 b \psi(b)$. Then

$$
\psi^{-1}\left(\varsigma_{l}^{*}\right) \sum_{1 \leq i \leq \lambda} \varsigma_{i}^{*} \leq c / 4, \quad 1 \leq l \leq n
$$

and so we have

$$
\begin{aligned}
(\mathrm{I}) & \leq P\left\{\sup _{a>0} \psi^{-1}(a) \sum_{i=1}^{n} \varsigma_{i}^{*} I\left[\varsigma_{i}^{*}>a\right]>c / 2\right\} \\
& \leq P\left\{\max _{1 \leq l \leq n} \sup _{\zeta_{i+1}^{*}<a \leq \varsigma_{i}^{*}} \psi^{-1}(a) \sum_{i=1}^{n} \varsigma_{i}^{*} I_{\left[\varsigma_{i}^{*}>a\right]}>c / 2\right\} \\
& \leq P\left\{\max _{1 \leq l \leq n} \psi^{-1}\left(\varsigma_{l}^{*}\right) \sum_{\lambda<i \leq l} \varsigma_{i}^{*}>c / 4\right\} \\
& \leq P\left\{\max _{\lambda<l \leq n}\left[\left(\sum_{\lambda<i \leq l} h_{i}\right) \psi^{-1}\left(\varsigma_{l}^{*}\right) \max _{\lambda<i \leq l} h_{i}^{-1} \varsigma_{i}^{*}\right]>c / 4\right\} \\
& \leq P\left\{\max _{\lambda<l \leq n} \varsigma_{l}^{*} / h_{l}>1\right\}+I_{\left[\max _{\lambda<l \leq n}\left\{\psi^{-1}\left(h_{l}\right) \sum_{\lambda<i \leq l} h_{i}\right\}>c / 4\right]} .
\end{aligned}
$$

Now, $\lambda \geq r$ by (iv), so that (ii) and (iii) imply

$$
\max _{\lambda<l \leq n} \psi^{-1}\left(h_{l}\right) \sum_{\lambda<i \leq l} h_{i} \leq \max _{r \leq l \leq n} \psi^{-1}\left(h_{l}\right) \sum_{i=r}^{l} h_{i} \leq 1 .
$$


Hence, the above indicator is zero $(c>4 d>4)$. We apply inequality $(1.2)$ to obtain

$$
\begin{aligned}
(\mathrm{I}) & \leq P\left\{\max _{\lambda<l \leq n} \varsigma_{l}^{*} / h_{l}>1\right\} \\
& \leq \sum_{\lambda<l \leq n} P\left\{\sum_{j=1}^{n} I_{\left[\varsigma_{j}>h_{l}\right]}>l\right\} \\
& \leq \sum_{\lambda<l \leq n}\left(e \sum_{j=1}^{n} P\left\{\varsigma_{j} \geq h_{l}\right\} / l\right)^{l} \leq \sum_{\lambda<l \leq n}(e / 3)^{l} \\
& \leq \frac{3}{3-e} \exp \left\{-\frac{c \ln (3 / e)}{4 b \psi(b)}\right\}<11 \exp \left\{-\frac{c \ln (3 / e)}{4 b \psi(b)}\right\} .
\end{aligned}
$$

Now (3.1) follows from the estimates (3.2), (3.3) and (3.4).

Examples of sequences $\left\{h_{l}\right\}$ and functions $\psi$ are

(1) $h_{l}=l^{-1 / p}, \psi(x)=(p x /(p-1))^{1 /(p-1)}, l \in \mathbf{N}, p \in(1, \infty), x>0$.

(2) $h_{l}=l^{-1}, \psi(x)=e / x e^{1 / x}, l \in \mathbf{N}, 0<x \leq 1$.

See 4.1 and 5.1 for additional examples.

4. The central limit theorem, non-i.i.d. case. In the next theorem, the function $\varphi: \mathbf{R}^{+} \rightarrow \mathbf{R}^{+}$and the constants $h_{k}, k \in \mathbf{N}$, will satisfy the following properties:

$$
\begin{gathered}
\varphi(x) \uparrow \infty, \quad x / \varphi(x) \uparrow \infty \quad \text { as } x \uparrow \infty, \text { and } \\
\varphi(x) \downarrow 0, \quad x / \varphi(x) \downarrow 0 \quad \text { as } x \downarrow 0, \\
\int_{0} \varphi\left(\ln x^{-1}\right) d x<\infty, \\
h_{k} \downarrow 0 \quad \text { as } k \uparrow \infty,
\end{gathered}
$$

and

there exist constants $K>0$ and $L>1$ such that

(i) $h_{l} \varphi^{-1}\left(\sum_{i=1}^{l} h_{i}\right) \leq \sum_{i=1}^{l} h_{i}, \quad l \geq L$;

(ii) $\quad h_{l} \leq K h_{l+1}, \quad l \geq L$;

(iii)

$$
\sum_{i=l}^{\infty} h_{i}^{2} \leq K h_{l} \sum_{i=1}^{l} h_{i}, \quad l \geq L
$$

(and we can also assume, by changing $K$ if necessary, that

$$
\left.l h_{l} \leq K \sum_{i=1}^{l} h_{i}, \quad l \geq L\right)
$$

4.1. EXAMPLES. (1) Let $H(x), x>0$, be regularly varying at infinity with exponent $1 / p, p \in(1,2)$ and such that

$$
H(x) \downarrow 0 \text { and } \quad x / H(x) \downarrow 0 \quad \text { as } x \downarrow 0,
$$

$H(x) \uparrow \infty$ and $\quad x / H(x) \uparrow \infty \quad$ as $x \uparrow \infty, \quad$ and $\quad \int_{0}^{1} d x / H(x)<\infty$. 
Then $h_{k}=2 p^{\prime} / H(k)$ and $\varphi(x)=x / H(x)$ satisfy (4.1)-(4.4) for suitable $L$ and $K$ because of the well-known limits (Feller (1971)):

$$
\lim _{x \rightarrow \infty} x^{-1}\left[H(x) \int_{0}^{x} \frac{d t}{H(t)}\right]=p^{\prime}, \quad \text { where } 1 / p+1 / p^{\prime}=1,
$$

and

$$
\lim _{x \rightarrow \infty} x^{-1}\left[(H(x))^{2} \int_{x}^{\infty} \frac{d t}{(H(t))^{2}}\right]=\frac{p}{(2-p)} .
$$

(2) $\varphi(x)=\ln x$ for $x \geq e, \varphi(x)=x^{2} / e^{2}$ for $0<x \leq e ; h_{l}=1 / l, l \in \mathbf{N}$.

These examples include those at the end of $\S 3$.

The following is the main result of this paper:

4.2. THEOREM. Let $\varphi(x), x \in \mathbf{R}^{+}$and $h_{k}, k \in \mathbf{N}$, satisfy the properties (4.1) to (4.4), and let $b_{n}, n \in \mathbf{N}$, be positive finite constants. Let $\mathscr{F} \subset \mathscr{L}_{0}(S, \mathscr{S})$ with envelope $F(s)$ finite for all $s \in S$. Let $\left\{X_{n j}: j=1, \ldots, n, n \in \mathbf{N}\right\}$ be a triangular array of row-wise independent $S$-valued random variables and for each $n$ let $\left\{\varepsilon_{n j}: j=1, \ldots, n\right\}$ be a Rademacher sequence independent of the $X_{n j}, j=$ $1, \ldots, n$. Assume:

(i) The sequence of set functions

$$
\left\{\left.\sum_{j=1}^{n} \mathscr{L}^{*}\left(\left\{f\left(X_{n j}\right) / b_{n}: f \in \mathscr{F}\right\}\right)\right|_{\left[\|x\|_{\mathcal{F}}>\delta\right]}\right\}_{n=1}^{\infty}
$$

is eventually tight in $l^{\infty}(\mathscr{F})$.

(ii) There is a pseudo distance $\rho$ on $\mathscr{F}$ and a Borel probability measure $\mu$ on $(\mathscr{F}, \rho)$ such that

(iia) $\lim _{\delta \rightarrow 0} \sup _{f \in \mathscr{F}} \int_{0}^{\delta} \varphi\left(\ln 1 / \mu\left\{B_{\rho}(f, \varepsilon)\right\}\right) d \varepsilon=0$ with $\sup _{f \in \mathscr{F}}$ finite for $\delta=$ $\infty$, and

(iib) there are constants $\sigma>0, n_{0}>0$ and $L_{1} \geq 1$ such that for all $f \in \mathscr{F}$, $l \geq L_{1}, n \geq n_{0}$ and $\varepsilon>0$,

$$
\sum_{j=1}^{n} P_{n j}^{*}\left\{\varepsilon^{-1} \sup _{g \in B_{\rho}(f, \varepsilon)}|f-g| / b_{n}>\sigma h_{l}\right\} \leq l / 3,
$$

where $P_{n j}=\mathscr{L}\left(X_{n j}\right)$.

(iii) For each $k \in \mathbf{N}$ and $f_{1}, \ldots, f_{k} \in \mathscr{F}$, the triangular array of $\mathbf{R}^{k}$-valued random variables $\left\{\left(f_{1}\left(X_{n j}\right), \ldots, f_{k}\left(X_{n j}\right)\right) / b_{n}: j=1, \ldots, n, n \in \mathbf{N}\right\}$ is infinitesimal and the sequence

$$
\left\{\sum_{j=1}^{n} \varepsilon_{n j}\left(f_{1}\left(X_{n j}\right), \ldots, f_{k}\left(X_{n j}\right)\right) / b_{n}\right\}_{n=1}^{\infty}
$$

converges in law to an infinitely divisible law with a degenerate Gaussian component.

Then, the sequence of random elements $\left\{\sum_{j=1}^{n} \varepsilon_{n j} f\left(X_{n j}\right) / b_{n}: f \in \mathscr{F}\right\}_{n=1}^{\infty}$ converges in law to a Radon infinitely divisible measure $\gamma$ on $l^{\infty}(\mathscr{F})$ with a degenerate Gaussian component. 
ProOF. By Corollary 2.2, convergence in law to a Radon probability measure of $l^{\infty}(\mathscr{F})$ will follow if we show

$$
\lim _{\delta \rightarrow 0} \limsup _{n} \operatorname{Pr}^{*}\left\{\left\|\sum_{j=1}^{n} \varepsilon_{n j} f I_{\left[F \leq \delta b_{n}\right]}\left(X_{n j}\right) / b_{n}\right\|_{\mathscr{F}}>\varepsilon\right\}=0
$$

for all $\varepsilon>0$. That the limit is infinitely divisible without Gaussian component follows then by condition (iii): since the limit $\gamma$ is Radon, it is supported by a separable Banach subspace of $l^{\infty}(\mathscr{F})$, and then $\gamma$ is infinitely divisible with only a degenerate Gaussian component by condition (iii) above, Theorem 7.9 of HoffmannJørgensen (1984) and Remark 5 there, with $\psi=\left\{\exp \left(i \sum_{\text {finite }} \alpha_{k} f_{k}\right): \alpha_{k} \in \mathbf{R}\right.$, $\left.f_{k} \in \mathscr{F}\right\}$ (where $\sum \alpha_{k} f_{k}(x):=\sum \alpha_{k} x\left(f_{k}\right), x \in l^{\infty}(\mathscr{F})$ ).

We now prove (4.6). As shown in Andersen et al. (1988, Lemma 2.1 and Remark 2.2(3)), by (4.1) and (4.2) there is no loss of generality in assuming that $\mu$ is discrete, with support $\left\{\pi_{q} f: q \in \mathbf{N}, f \in \mathscr{F}\right\} \subset \mathscr{F}, \#\left\{\pi_{q} f: f \in \mathscr{F}\right\}<\infty$ for all $q$, satisfying

$$
\left\{\begin{array}{l}
\rho\left(\pi_{q} f, f\right) \leq 2^{-q}, \quad q \in \mathbf{N}, f \in \mathscr{F}, \text { and } \\
\lim _{q_{0} \rightarrow \infty} \beta_{q_{0}}:=\lim _{q_{0} \rightarrow \infty} \sup _{f \in \mathscr{F}} \sum_{q \geq q_{0}} 2^{-q} \gamma_{q}(f)=0
\end{array}\right.
$$

with $\sup _{f}$ finite for $q_{0}=1$, and

$$
\gamma_{q}(f):=\varphi\left(\ln \frac{2^{q}}{\mu\left\{\pi_{1} f\right\} \cdots \mu\left\{\pi_{q} f\right\}}\right) .
$$

Letting, for each $q \in \mathbf{N}, T_{q}:=\left\{\left(\pi_{1} f, \ldots, \pi_{q} f\right): f \in \mathscr{F}\right\}$ it is obvious that $\gamma_{q}$ is actually defined on $T_{q}$, so that we write $\gamma_{q}(t)=\gamma_{q}(f)$ if $t=\left(\pi_{1} f, \ldots, \pi_{q} f\right)$. Then, by Andersen et al. (1988, Lemma 2.4), we have

$$
\lim _{r \rightarrow \infty} \sum_{q=1}^{\infty} \sum_{t \in T_{q}} \exp \left\{-r \varphi^{-1}\left(s \gamma_{q}(t)\right)\right\}=0
$$

for every $s>0$, with the sum finite for every $r>1$. Now let $\psi(x):=1 / x \varphi^{-1}(1 / x)$ $\left(\uparrow \infty\right.$ as $x \uparrow \infty, \downarrow 0$ as $x \downarrow 0$ by (4.1)). For $n, q, q_{0}, f$, define

$$
\begin{aligned}
c_{n, q}(f) & =2^{-q} b_{n} \psi\left(1 / \gamma_{q} f\right), \\
\Delta_{q}^{j}(f) & =\min _{1 \leq r \leq q}\left[\sup _{g \in B_{\rho}\left(\pi_{r} f, 3 / 2^{-r}\right)}\left|g-\pi_{r} f\right|\right]^{*},
\end{aligned}
$$

where the ${ }^{*}$ denotes the outer measurable envelope with respect to $P_{n j}:=\mathscr{L}\left(X_{n j}\right)$, and

$$
\tau_{j}(f)=\min \left\{q \geq q_{0}: \Delta_{q}^{j}(f)>c_{n, q+1}(f)\right\}, \quad j=1 \ldots n .
$$

(Sometimes we write $\gamma_{q} f, c_{n, q} f, \Delta_{q}^{j} f, \tau_{j} f$ instead of $\gamma_{q}(f), c_{n, q}(f), \Delta_{q}^{j}(f)$ and $\tau_{j}(f)$ respectively.) Note that

$$
\begin{gathered}
\varphi(q \ln 2) \leq \gamma_{q}(f)=o\left(2^{q}\right) \quad \text { and } \quad \gamma_{q}(f) \uparrow \infty \quad \text { as } q \rightarrow \infty, \\
\inf _{f \in \mathscr{F}} \psi\left(1 / \gamma_{q} f\right)>0 \quad \text { for all } q>0, \\
\Delta_{q}^{j}(f) \downarrow \quad \text { as } q \uparrow \quad \text { for all } f \text { and } j,
\end{gathered}
$$




$$
\left|f-\pi_{q} f\right| \leq 2 \Delta_{q}^{j}(f), \quad\left|\pi_{q} f-\pi_{q-1} f\right| \leq 2 \Delta_{q}^{j}(f) .
$$

By summation by parts, as in Andersen et al. (1988), we have for all $f \in \mathscr{F}$ and $q_{0}<q_{1}$,

$$
\begin{aligned}
f= & \pi_{q_{0}} f+\left(f-\pi_{q_{0}} f\right) I_{\left[\tau f=q_{0}\right]}+\left(f-\pi_{q_{1}} f\right) I_{\left[\tau f \geq q_{1}\right]} \\
& +\sum_{q=q_{0}+1}^{q_{1}-1}\left(f-\pi_{q} f\right) I_{[\tau f=q]}+\sum_{q=q_{0}+1}^{q_{1}}\left(\pi_{q} f-\pi_{q-1} f\right) I_{[\tau f \geq q]} .
\end{aligned}
$$

Therefore,

$$
\begin{aligned}
\operatorname{Pr}^{*}\{ & \left.\left\|\sum_{j=1}^{n} \varepsilon_{n j} f I_{\left[F \leq \delta b_{n}\right]}\left(X_{n j}\right)\right\|_{\mathscr{F}}>6 \varepsilon b_{n}\right\} \\
\leq & \operatorname{Pr}\left\{\left\|\sum_{j=1}^{n} \varepsilon_{n j}\left(\pi_{q_{0}} f\right) I_{\left[F \leq \delta b_{n}\right]}\left(X_{n j}\right)\right\|_{\mathscr{F}}>\varepsilon b_{n}\right\} \\
& +\operatorname{Pr}^{*}\left\{\left\|\sum_{j=1}^{n} \varepsilon_{n j}\left(f-\pi_{q_{0}} f\right) I_{\left[\tau_{j} f=q_{0}\right]} I_{\left[F \leq \delta b_{n}\right]}\left(X_{n j}\right)\right\|_{\mathscr{F}}>\varepsilon b_{n}\right\} \\
& +\operatorname{Pr}^{*}\left\{\left\|\sum_{j=1}^{n} \varepsilon_{n j}\left(f-\pi_{q_{1}} f\right) I_{\left[\tau_{j} f \geq q_{1}\right]} I_{\left[F \leq \delta b_{n}\right]}\left(X_{n j}\right)\right\|_{\mathscr{F}}>\varepsilon b_{n}\right\} \\
& +\operatorname{Pr}\left\{\left\|\sum_{q=q_{0}+1}^{q_{1}-1} \sum_{j=1}^{n}\left(\Delta_{q}^{j} f\right) I_{\left[c_{n, q+1} f<\Delta_{q}^{j} f \leq c_{n, q} f\right]}\left(X_{n j}\right)\right\|_{\mathscr{F}}>\varepsilon b_{n}\right\} \\
& +\operatorname{Pr}^{*}\left\{\left\|\sum_{q=q_{0}+1}^{q_{1}} \sum_{j=1}^{n} \varepsilon_{n j}\left(\pi_{q} f-\pi_{q-1} f\right) I_{\left[\tau_{j} f \geq q\right]} I_{\left[F \leq \delta b_{n}\right]}\left(X_{n j}\right)\right\|_{\mathscr{F}}>\varepsilon b_{n}\right\} \\
:= & (\mathrm{I})+(\mathrm{II})+(\mathrm{III})+(\mathrm{IV})+(\mathrm{V}) .
\end{aligned}
$$

Now,

$$
\lim _{\delta \rightarrow 0} \limsup _{n}(\mathrm{I})=0 \quad \text { for all } q_{0}<\infty
$$

by the CLT in finite dimensions, on account of condition (iii). To handle (II) we note that for $\delta$ small enough and $q_{0} \geq 1$ fixed, we have by (4.11), $\inf _{f} c_{n, q_{0}+1} f / b_{n}>2 \delta$. Furthermore, by definition, $\left|\Delta_{q_{0}}^{j} f\right| \leq 2 F$. Hence,

$$
\left\{\tau_{j} f=q_{0}\right\} \cap\left\{F \leq \delta b_{n}\right\}=\varnothing .
$$

Therefore, for such $\delta$ 's, (II) $=0$, that is,

$$
\lim _{\delta \rightarrow 0} \limsup _{n}(\mathrm{II})=0 .
$$

By (4.1) and (4.10) $\sup _{f \in \mathscr{F}} \psi\left(1 / \gamma_{q} f\right) \rightarrow 0$ as $q \rightarrow \infty$. Hence $n\left(\sup _{f \in \mathscr{F}} c_{n, q} f\right)=$ $n b_{n} 2^{-q} O(1)$ and we can choose $q_{1}(n)$ such that $n\left(\sup _{f \in \mathscr{F}} c_{n, q_{1}(n)} f\right)<\varepsilon b_{n}$ (e.g., $q_{1}(n) \sim \ln n$ ). For such $q_{1}$ we obviously have

$$
\text { (III) }=0 \text {. }
$$


To estimate (IV) we make use of Theorem 3.1. With $t_{q} f=\left(\pi_{1} f, \ldots, \pi_{q} f\right)$ and taking into account that $\Delta_{q}^{j} f, \gamma_{q} f$ and $c_{n q} f$ depend on $f$ only through $t_{q} f$ we have

$$
\begin{gathered}
(\mathrm{IV}) \leq \sum_{q=q_{0}+1}^{q_{1}-1} \sum_{t \in T_{q}} \operatorname{Pr}^{*}\left\{\sup _{\left\{f: t_{q} f=t\right\}}\left(\gamma_{q+1} f\right)^{-1} \sum_{j=1}^{n} b_{n}^{-1} 2^{q+1} \Delta_{q}^{j} f\right. \\
\left.\times I_{\left[c_{n, q+1} f<\Delta_{q}^{j} f<c_{n q} f\right]}\left(X_{n j}\right)>\varepsilon \beta_{q_{0}+2}^{-1}\right\} \\
\leq \sum_{q=q_{0}+1} \sum_{t \in T_{q}} \operatorname{Pr}^{*}\left\{\sup _{0<a<1 / \gamma_{q} t} a \cdot \sum_{j=1}^{n} 2^{q+1} b_{n}^{-1} \Delta_{q}^{j} f\right. \\
\left.\times I_{\left[\psi(a)<2^{q+1} b_{n}^{-1} \Delta_{q}^{j} f \leq 2 \psi\left(1 / \gamma_{q} f\right)\right]}\left(X_{n j}\right)>\varepsilon \beta_{q_{0}+2}^{-1}\right\} .
\end{gathered}
$$

We want to use Theorem 3.1 with $\xi_{i}=2^{q+1} b_{n}^{-1} \Delta_{q}^{j} f, b=1 / \gamma_{q} t, d=2, c=\varepsilon \beta_{q_{0}+2}^{-1}$ and $r=L \vee L_{1}$ (see (4.4) and (iib)). Since in (4.5) we may assume $\sigma=1 / 6$ just by multiplying either $\rho$ or $b_{n}$ by a suitable constant, then (4.5) implies (i) in Theorem 3.1. We can choose $q_{0}$ large enough such that $c>8, h_{r} \geq \psi\left(1 / \gamma_{q} t\right)$ and $4 r\left(\gamma_{q} t\right)^{-1} \psi\left(1 / \gamma_{q} t\right) \leq c$ for $q \geq q_{0}$ and $t \in T_{q}$, so that (iii) and (iv) in 3.1 hold too. Finally, (4.4)(i) is just (ii) in Theorem 3.1. We thus obtain

$$
(\mathrm{IV}) \leq \sum_{q=q_{0}+1}^{q_{1}-1} \sum_{t \in T_{q}} 12 \cdot \exp \left\{-\frac{\varepsilon \ln (3 / e) \varphi^{-1}\left(\gamma_{q} t\right)}{4 \cdot \beta_{q_{0}+1}}\right\} \underset{q_{0} \rightarrow \infty}{\longrightarrow} 0
$$

uniformly in $n, \delta$ and $q_{1}$.

We finally estimate (V) using Bernstein's inequality as in Andersen et al. (loc. cit.):

$$
(\mathrm{V}) \leq 2 \sum_{q=q_{0}+1}^{q_{1}} \sum_{t \in T_{q}} \exp \left\{\frac{-\left(\varepsilon / \beta_{q_{0}+1}\right)^{2} 2^{-2 q}\left(\gamma_{q} t\right)^{2} b_{n}^{2}}{8 \cdot \sum_{j=1}^{n} P_{n j}\left(\Delta_{q}^{j} t\right)^{2} I_{\left[\Delta_{q}^{j} t \leq c_{n, q} t\right]}+2^{-q+1}\left(\gamma_{q} t\right) \varepsilon\left(c_{n, q} t\right) b_{n} / 3 \beta_{q_{0}+1}}\right\} .
$$

Letting $\eta_{j}=2^{q} b_{n}^{-1} \Delta_{q}^{j} t I_{\left[\Delta_{q}^{j} t \leq c_{n, q} t\right]}\left(X_{n, j}\right)$, we have $\sum_{j=1}^{n} P\left(\eta_{j}>h_{l}\right) \leq l / 3$. Let $l_{1}=l_{1}(q)$ be defined by the inequality

$$
h_{l_{1}+1}<\psi\left(1 / \gamma_{q} t\right) \leq h_{l_{1}}
$$


for $q \geq q_{0}$ ( $q_{0}$ is chosen large enough so that $l_{1}(q) \geq L_{1} \vee L$, for all $q \geq q_{0}$ ). Then, using the properties in (4.4), we have

$$
\begin{aligned}
\sum_{j=1}^{n} P_{n j}\left(\Delta_{q}^{n} t\right)^{2} I_{\left[\Delta_{q}^{j} t \leq c_{n, q} t\right]}=2^{-2 q} b_{n}^{2} \sum_{j=1}^{n} E \eta_{j}^{2} I_{\left[\eta_{j} \leq \psi\left(1 / \gamma_{q} t\right)\right]} \\
\quad \leq 2 \cdot 2^{-2 q} b_{n}^{2} \sum_{j=1}^{n} \int_{0}^{\psi\left(1 / \gamma_{q} t\right)} u P\left\{\eta_{j}>u\right\} d u \\
\quad \leq 2^{-2 q+1} b_{n}^{2} \sum_{j=1}^{n} \sum_{l=l_{1}(q)}^{\infty} \int_{h_{l+1}}^{h_{l}} u P\left\{\eta_{j}>u\right\} d u \\
\quad \leq 2^{-2 q} b_{n}^{2} \sum_{l=l_{1}(q)}^{\infty}(l+1)\left(h_{l}^{2}-h_{l+1}^{2}\right) \\
\quad=2^{-2 q} b_{n}^{2}\left[l_{1}(q) h_{l_{1}(q)}^{2}+\sum_{l=l_{1}(q)}^{\infty} h_{l}^{2}\right] \\
\quad \leq 2^{-2 q+1} b_{n}^{2} K h_{l_{1}(q)} \sum_{l=1}^{l_{1}(q)} h_{l} \\
\leq 2^{-2 q+1} b_{n}^{2} K^{2} h_{l_{1}(q)+1} \sum_{l=1}^{l_{1}(q)} h_{l} \\
\leq 2^{-2 q+1} b_{n}^{2} K^{2} \psi\left(1 / \gamma_{q} t\right) \gamma_{q} t=2^{-2 q+1} b_{n}^{2} K^{2}\left(\gamma_{q} t\right)^{2} / \varphi^{-1}\left(\gamma_{q} t\right)
\end{aligned}
$$

where in the last inequality we have used that

$$
\psi\left(1 / \gamma_{q} t\right) \leq h_{l_{1}(q)} \leq \psi\left(1 / \sum_{i=1}^{l_{1}(q)} h_{i}\right) \quad(\text { by }(4.4)(\mathrm{i}))
$$

i.e. since $\psi(x)$ is nondecreasing, $\gamma_{q} t \geq \sum_{i=1}^{l_{1}(q)} h_{i}$. Using (4.20) we get

$$
(\mathrm{V}) \leq 2 \sum_{q=q_{0}+1}^{q_{1}} \sum_{t \in T_{q}} \exp \left\{-\frac{\varepsilon^{2} \varphi^{-1}\left(\gamma_{q} t\right)}{\left(K^{\prime}+2 \varepsilon / \beta_{q_{0}+1}\right) \beta_{q_{0}+1}^{2}}\right\}
$$

where $K^{\prime}$ is a suitable constant (independent of $q, n, q_{1}, q_{0}$, etc.). Since $\beta_{q_{0}+1} \rightarrow 0$ we obtain by (4.9)

$$
\lim _{q_{0} \rightarrow \infty}(\mathrm{V})=0 \quad \text { uniformly in } n, q \text {, and } \delta .
$$

Now (4.15), (4.16), (4.17), (4.18) and (4.21) give

$$
\lim _{q_{0} \rightarrow \infty} \limsup _{\delta \rightarrow 0} \limsup _{n \rightarrow \infty}((\mathrm{I})+\cdots+(\mathrm{V}))=0,
$$

which by (4.14) is nothing but (4.6).

Regarding stochastic boundedness, we have

4.3. Proposition. Let $\varphi,\left\{h_{k}\right\}$ and $\left\{b_{n}\right\}$ be as in Theorem 4.2. Let $\mathscr{F} \subset$ $\mathscr{L}_{0}(S, \mathscr{S})$ have everywhere finite envelope $F$. Let $\left\{X_{n j}: j=1, \ldots, n \in \mathbf{N}\right\}$ be a 
triangular array of row-wise independent $S$-valued random variables and, for each $n$, $\left\{\varepsilon_{n j}: j=1, \ldots, n\right\}$ a Rademacher sequence independent of the $X_{n j}, j=1, \ldots, n$. Assume:

(i) $\lim _{M \rightarrow \infty} \sup _{n} \sum_{j=1}^{n} \operatorname{Pr}^{*}\left\{F\left(X_{n j}\right)>M b_{n}\right\}=0$.

(ii) For all $f \in \mathscr{F}, l>l_{0}, n>n_{0}$ and $\varepsilon>0$, and for some $\sigma>0, n_{0} \geq 1$ and $l_{0} \geq 1$,

$$
\sum_{j=1}^{n} P_{n j}^{*}\left\{\varepsilon^{-1} \sup _{g \in B_{\rho}(f, \varepsilon)}|f-g| / b_{n}>\sigma h_{l}\right\} \leq l / 3,
$$

where $\rho$ is a pseudodistance on $\mathscr{F}$ for which there exists a probability measure $\mu$ such that

$$
\sup _{f \in \mathscr{F}} \int_{o}^{\infty} \varphi\left(\ln \frac{1}{\mu\left\{B_{\rho}(f, \varepsilon)\right\}}\right) d \varepsilon<\infty
$$

Then the sequence $\left\{\sum_{j=1}^{n} \varepsilon_{n j} f\left(X_{n j}\right) / b_{n}: f \in \mathscr{F}\right\}_{n=1}^{\infty}$ is stochastically bounded.

We omit the proof, which is similar to that of Theorem 4.2 (and uses Remark 2.4 instead of Corollary 2.2; the main difference is that here

$$
\left.c_{n, q} f=M^{1 / 2} 2^{-q} b_{n} \psi\left(1 / \gamma_{q} f\right)\right) .
$$

In the next section we apply these two theorems to $p$-stable convergence for empirical processes, but they may be useful in other situations as well. Here is an easy example.

4.4. ExAmple. Let $U_{i}$ be independent uniform on $[0,1]$. Define $X_{n j}=n U_{j}$, $j=1, \ldots, n, n \in \mathbf{N}$. Let

$$
\mathscr{F}=\left\{f_{u}(x):=(u-x) I_{[x \leq u]}: u \in[0,1]\right\} .
$$

Then the sequence $\left\{\sum_{j=1}^{n} f_{u}\left(X_{n j}\right): u \in[0,1]\right\}$ converges weakly to a Radon measure of $l^{\infty}[0,1]$. Actually, the convergence takes place in $C[0,1]$. (Note that $f_{u}\left(n U_{j}\right)$ $=\int_{0}^{u} I_{\left[n U_{j} \leq t\right]} d t$ and it is clear that, since $\sum_{j=1}^{n} I_{\left[n U_{j} \leq t\right]}$ converges to the Poisson process-weakly in $D[0,1]$ - then $\sum_{j=1}^{n} \int_{0}^{u} I_{\left[n U_{j} \leq t\right]} d t$ must converge in law in $D$, hence in $C$, to the integrated Poisson processes.) We will check that this result follows easily from Theorem 4.2. Since $E \sum_{j=1}^{n} f_{u}\left(X_{n j}\right)=u^{2} / 2$, the same arguments given in $\S 2$ show that it is enough to consider the randomized version $\sum_{j=1}^{n} \varepsilon_{j} f_{u}\left(X_{n j}\right)$. To apply Theorem 4.2, let us check first condition (i): for all $\tau>0$

$$
\begin{aligned}
& \lim _{\varepsilon \rightarrow 0} \limsup _{n} n \lambda\left\{x: \sup _{|u-v|<\varepsilon, 0 \leq u, v \leq 1}\left|f_{u}(n x)-f_{v}(n x)\right|>\tau, \sup _{0 \leq u \leq 1} f_{u}(n x)>\delta\right\} \\
& \quad \leq \lim _{\varepsilon \rightarrow 0} \limsup _{n} n I_{[\varepsilon>\tau]}=0 \quad\left(\text { since }\left|f_{u}-f_{v}\right| \leq|u-v|\right),
\end{aligned}
$$

and

$$
\limsup _{n} n \lambda\left\{x: \sup _{0 \leq u \leq 1} f_{u}(n x)>\delta\right\}=1-\delta
$$


hence the sequence in (i) is eventually tight. As for (ii), we have many choices for $\varphi, \rho$ and $h_{k}$. Take $\rho(u, v)=|u-v|$. Then (4.5) is, for $\varepsilon \leq 1$ and $l \geq 6$,

$$
\begin{aligned}
\sup _{u \in[0,1]} & n \lambda\left\{\varepsilon^{-1}\left|f_{(u-\varepsilon) \vee 0}(n x)-f_{(u+\varepsilon) \wedge 1}(n x)\right|>\sigma h_{l}\right\} \\
\leq & \sup _{u \in[0,1]} n \lambda\left\{\left|f_{(u-\varepsilon) \vee 0}(n x)-f_{(u+\varepsilon) \wedge 1}(n x)\right|>0\right\} \\
\leq & \sup _{u \in[0,1]}(u+\varepsilon)<2 \leq l / 3 .
\end{aligned}
$$

Now, take e.g. $h_{l}=l^{-1 / p}, \varphi(x)=x^{1 / p^{\prime}}$ for any $p \in(1,2)$ and $\mu=$ Lebesgue measure on $[0,1]$.

4.5. EXAMPLE. Theorem 4.2 can be used to obtain weak convergence of the processes considered in Csörgö et al. $(1986, \S 3)$. We only indicate how to check conditions (i) and (ii) of Theorem 4.2 for the processes $\left\{\sum_{i=1}^{n} \varepsilon_{i} f_{t}\left(n U_{i}\right): t \geq 0\right\}$ where $f_{0}(x)=0, f_{t}(x):=p^{-1} \int_{0}^{t} I_{[x \leq s]} d s / s^{1+1 / p}, x \in(0, \infty)$, and $\left\{U_{i}\right\}_{i=1}^{\infty}$ are i.i.d. uniform on $(0,1)$. (Here $S=(0,1), \mathscr{S}=\mathscr{B}, X_{n j}=n U_{j}$ and $P_{n j}=\mathscr{L}\left(X_{n j}\right)$.) Just take $\delta>0$ such that $p<2(1-p \delta), h_{l}=l^{\delta-1 / p}, \varphi(x)=x^{\delta+1 / r^{\prime}}$, and $\rho(s, t)=\left|\int_{s}^{t} d x /(1+x)^{1+\delta}\right|, s, t \geq 0$.

5. The CLT with stable limits. We start by applying Theorem 4.2 to the CLT for i.i.d. random variables $X_{i}$ and $p$-stable limits. We note that we cannot obtain a result for all normings $B(n)=n^{1 / p} M(n)$ with $M$ slowly varying at infinity, but only under a submultiplicativity property for $B$ which is essentially the same that Marcus (1986) used in a different, but related, context. The reason for this constraint appears explicitly in the proof of Theorem 5.2 that follows (and also in the proof of Theorem 3.1).

Let now $B(x)$ and $H(x), x \in[0, \infty)$, be real valued functions satisfying:

$$
B(u) H(v) \leq K B(u v) \text { for some } K>0, u \geq 0, v \geq 1
$$

$B$ and $H$ are regularly varying functions with exponent $1 / p$

for some $p \in(1,2)$, continuous, strictly increasing,

$$
B(0)=H(0)=0 \text { and such that } \int_{0}^{1} d t / H(t)<\infty ;
$$

the function $\varphi(x):=x / H(x), x>0, \varphi(0):=0$ is strictly

increasing and continuous.

5.1. EXAMPLES. The following subsets of the set of examples in 4.1 satisfy (5.1)-(5.3). Let $L x:=\ln (e \vee x)$.

(1) $B(x)=H(x)=x^{1 / p} /(L x)^{\alpha}, \alpha \geq 0,1<p<2$, and then $\varphi(x) \simeq x^{1 / p^{\prime}}(L x)^{\alpha}$ where $1 / p+1 / p^{\prime}=1$.

(2) $B(x)=x^{1 / p}(L x)^{\alpha}, \alpha>0,1<p<2$, and $H(x)=x^{1 / p}, \varphi(x)=x^{1 / p^{\prime}}$.

Theorem 4.2 gives the following corollary for stable limits:

5.2. THEOREM. Let $p \in(1,2)$ and let $B, H, \varphi$ be functions satisfying (5.1) to (5.3) for this value of $p$. Let $\mathscr{F} \subset \mathscr{L}_{1}(S, \mathscr{S}, P)$ with $F(x)<\infty$ for all $s \in S$. Let 
$\left\{X_{i}\right\}_{i=1}^{\infty}$ be i.i.d. $S$-valued random variables with law $P$. Assume:

(i) The finite dimensional distributions of the sequence

$$
\left\{\sum_{i=1}^{n}\left(f\left(X_{i}\right)-P f\right) / B(n): f \in \mathscr{F}\right\}_{n=1}^{\infty}
$$

converge in law (necessarily to $p$-stable measures in Euclidean space);

(ii) The sequence of set functions on $l^{\infty}(\mathscr{F})$,

$$
\left\{\left.n \mathscr{L}^{*}\left(\delta_{X_{1}} / B(n)\right)\right|_{\left[\|x\|_{,}>\delta\right]}\right\}_{n=1}^{\infty}
$$

is eventually tight for all $\delta>0$, and

(iii) There are a pseudodistance $\rho$ on $\mathscr{F}$ and a Borel probability measure $\mu$ on $(\mathscr{F}, \rho)$ such that

(iiia) $\lim _{\delta \rightarrow 0} \sup _{f \in \mathscr{F}} \int_{0}^{\delta} \varphi\left(\ln 1 / \mu\left\{B_{\rho}(f, \varepsilon)\right\}\right) d \varepsilon=0$ with $\sup _{f \in \mathscr{F}}$ finite for $\delta=$ $\infty$, and

(iiib) There exists $c<\infty$ such that for all $f \in \mathscr{F}$ and $\varepsilon>0$

$$
\sup _{t>0} t P\left\{\varepsilon^{-1}\left[\sup _{g \in B_{\rho}(f, \varepsilon)}|f-g|\right]^{*}>B(t)\right\} \leq c
$$

Then, the sequence (5.4) converges in law in $l^{\infty}(\mathscr{F})$ to a $p$-stable Radon measure, i.e. $\mathscr{F} \in \operatorname{CLT}(P, B(n))$.

Proof. By taking $B(3 c t)$ instead of $B(t)$ if necessary, we may assume $c$ in (5.6) to be $1 / 3$. Now, since $B(t) / H(l) \geq B(t / l) / K$ for all $l \geq 1,(5.6)$ implies (4.5) with $b_{n}=B(n), h_{l}=1 / H(l), \sigma=K$ and $P_{n j}=P$. Also, (4.1) to (4.3) follow from the well-known properties of regularly varying functions (see Example 4.1 ); to obtain (4.4) it may be necessary to replace $H$ by a suitable multiple. So, the proof of Theorem 4.2 applies to give

$$
\lim _{\delta \rightarrow 0} \limsup _{n} \operatorname{Pr}^{*}\left\{\left\|\sum_{j=1}^{n} \varepsilon_{j} f I_{[F \leq \delta B(n)]}\left(X_{j}\right) / B(n)\right\|_{\mathscr{F}}>\varepsilon\right\}=0
$$

for all $\varepsilon>0$, and this is enough to prove Theorem 5.2 in view of Proposition 2.5.

5.3. REMARKS. (1) Talagrand's (1986) recent result on necessary conditions for sample continuity of stable measures allows for the replacement of condition (iiia) on $\rho$ and $\mu$ by

(iiia) $\rho$ is the $L_{1}$ (or equivalently $\Lambda_{p, \infty}$ ) pseudodistance associated to a Radon $p$-stable probability measure on $l^{\infty}(\mathscr{F})$.

But (iiia)' is a stronger condition than (iiia), in contrast with the Gaussian case (Theorems 4.1 and 4.4 in Andersen et al. (1988)).

(2) Conditions (ii) and (iiib) in the previous theorem can be replaced by the stronger condition: there exists $c<\infty$ such that for all $\varepsilon>0$,

$$
\sup _{t>0} t P\left\{\varepsilon^{-1}\left[\sup _{\{f, g \in \mathcal{F}: \rho(f, g) \leq \varepsilon\}}|f-g|\right]^{*}>B(t)\right\} \leq c .
$$

Since $(\mathscr{F}, \rho)$ is totally bounded, (5.7) implies that the set functions (5.5) are uniformly bounded; moreover (5.7) expresses the fact that the sequence of set functions (5.5) is asymptotically $\rho$-equicontinuous, hence the sequence (5.5) is eventually tight. 
We state next a result for the case $p=1, B(n)=n$ and randomized empiricals (these limitations are imposed only in order to avoid technicalities such as complicated expressions for $\varphi$ and/or truncated expectations). It is an immediate consequence of Theorem 4.2 (see Example 4.1(2)).

5.4. THEOREM. Let $\mathscr{F} \subset \mathscr{L}_{0}(S, \mathscr{S}, P)$, with $F(s)<\infty$ for all $s \in S$. Assume, for $\left\{X_{i}\right\}_{i=1}^{\infty}$ i.i.d. $S$-valued random variables with law $P$ and $\left\{\varepsilon_{i}\right\}_{i=1}^{\infty} a$ Rademacher sequence independent of $\left\{X_{i}\right\}_{i=1}^{\infty}$ :

(i) The finite dimensional distributions of the sequence

$$
\left\{\sum_{j=1}^{n} \varepsilon_{j} f\left(X_{j}\right) / n: f \in \mathscr{F}\right\}
$$

converge in law.

(ii) The sequence of set functions on $l^{\infty}(\mathscr{F})$,

$$
\left\{\left.n \mathscr{L}^{*}\left(\delta_{X_{1}} / n\right)\right|_{\left[\|x\|_{\mathscr{F}}>\delta\right]}\right\}_{n=1}^{\infty}
$$

is eventually tight for all $\delta>0$.

(iii) There are a pseudodistance $\rho$ on $\mathscr{F}$ and a Borel probability measure $\mu$ on $(\mathscr{F}, \rho)$ such that

(iiia) $\lim _{\delta \rightarrow 0} \sup _{f \in \mathscr{F}} \int_{0}^{\delta} \ln _{+}\left(\ln 1 / \mu\left\{B_{\rho}(f, \varepsilon)\right\}\right) d \varepsilon=0$, with $\sup _{f \in \mathscr{F}}$ finite for $\delta=$ $\infty$, and

(iiib) There exists $c>0$ such that for all $f \in \mathscr{F}$ and $\varepsilon>0$,

$$
\sup _{t>0} t P^{*}\left\{\sup _{g \in B_{\rho}(f, \varepsilon)}|f-g|>t\right\} \leq c \varepsilon .
$$

Then, the sequence (5.8) converges in law in $l^{\infty}(\mathscr{F})$ to a 1-stable Radon measure.

5.5. REMARK. Remark 5.3 with $B(n)=n$ obviously applies also in the case of Theorem 5.4 .

5.6. EXAMPLE. A $C L T$ in $C(T)$. Proceeding as in the proof of Theorem 4.5 of Andersen et al. (1988) we obtain the following slight generalization of the CLT in Marcus and Pisier (1984, Corollary 1.4) (see also Juknevičienè (1986)) as a corollary of the preceding Theorems 5.2 and 5.4: Let $(T, d)$ be a compact metric or pseudometric space, and let $\{X(t), t \in T\}$ be a sample continuous process on $(T, d)$, centered for $1<p<2$ and symmetric for $p=1$. Let $B_{p}(x)$ and $\varphi_{p}(x)$ satisfy conditions (5.1)-(5.3) for $1<p<2$, and let $B_{1}(x)=x, \varphi_{1}(x)=\ln _{+} x$ for $p=1$. Assume, for $X_{i}$ i.i.d. with $\mathscr{L}\left(X_{i}\right)=\mathscr{L}(X)$,

(i) the finite dimensional distributions of

$$
\left\{\sum_{i=1}^{n} X_{i} / B_{p}(n)\right\}_{n=1}^{\infty}
$$

converge in law.

(ii) For all $\varepsilon>0, \tau>0$,

$$
\lim _{\delta \rightarrow 0} \limsup _{n \rightarrow \infty} n P\left\{\sup _{d(s, t)<\delta}|X(t)-X(s)|>\varepsilon B_{p}(u),\|X\|_{\infty}>\tau B_{p}(n)\right\}=0 .
$$


(iii) There exist a continuous pseudodistance $\rho$ on $T$ and a Borel probability measure $\mu$ on $(T, d)$ such that: and

(iiia) $\lim _{\delta \rightarrow 0} \sup _{t \in T} \int_{0}^{\delta} \varphi_{p}\left(\ln 1 / \mu\left\{B_{\rho}(t, \varepsilon)\right\}\right) d \varepsilon=0$ with $\sup _{t \in T}$ finite for $\delta=\infty$,

(iiib) there exists $c<\infty$ such that for all $\varepsilon>0$,

$$
\sup _{u>0} u P\left\{\sup _{\rho(s, t) \leq \varepsilon}|X(t)-X(s)| / \varepsilon>B(u)\right\} \leq c .
$$

Then the sequence (5.9) converges in law in $C(T)$ to the law of a $p$-stable sample continuous process.

5.6. REMARK. As a consequence of Theorem 4.3, we can drop conditions (i) and (ii) and weaken condition (iii) in Theorems 5.2 and 5.3, and still obtain stochastic boundedness, that is: Let $p \in(1,2)$ (resp. $p=1$ ) and let $B, H, \varphi$ satisfy (5.1) to (5.3) (resp. $B(n)=H(n)=n$ and $\left.\varphi(x)=\ln _{+} x\right)$, let $\mathscr{F} \subset \mathscr{L}_{1}(S, \mathscr{S}, P)$ (resp. $\mathscr{L}_{0}(S, \mathscr{S}, P)$ ) with $F$ everywhere finite, and let $\left\{X_{i}\right\}_{i=1}^{\infty}$ be i.i.d. $S$-valued random variables with law $P$ (and $\left\{\varepsilon_{i}\right\}_{i=1}^{\infty}$ a Rademacher sequence independent of $\left\{X_{i}\right\}_{i=1}^{\infty}$ in case $p=1$ ). Then, if there exist a pseudodistance $\rho$ on $\mathscr{F}$ and a probability measure $\mu$ on $(\mathscr{F}, \rho)$ such that condition (iii) of Theorem 5.2 (resp. Theorem 5.4) holds with (iiia) weakened to only

$$
\sup _{f \in \mathscr{F}} \int_{0}^{\infty} \varphi\left(\ln \frac{1}{\mu\left\{B_{\rho}(f, \varepsilon)\right\}}\right) d \varepsilon<\infty
$$

the sequence (5.4) (resp. (5.8)) is stochastically bounded.

Theorems 5.2 and 5.4, together with standard arguments in de Acosta (1981) or in Kuelbs and Zinn (1979) about the equivalence of the weak and the strong law of large numbers in Banach spaces, give the following laws of large numbers:

5.7. PROPOSITION. Consider the situation of Theorem 5.2, without condition (i) and with condition (ii) replaced by

(ii) $t P^{*}\{F>B(t)\} \rightarrow 0$ as $t \rightarrow \infty$.

Then

$$
\left\|\sum_{j=1}^{n}\left(f\left(X_{j}\right)-P f\right) / B(n)\right\|_{\mathscr{F}} \rightarrow 0 \text { in } \mathrm{pr}^{*} .
$$

If (ii)' is replaced by

(ii)" $P\left(B^{-1}(F)\right)<\infty$

(or equivalently, by $F\left(X_{j}\right) / B(j) \rightarrow 0$ a.s.), then

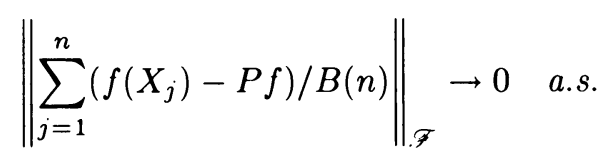

5.8. PROPOSITION. Let $\mathscr{F} \subset \mathscr{L}_{1}(S, \mathscr{P}, P)$ with $F$ everywhere finite and $\sup _{f \in \mathscr{F}}\left|P f I_{[|f|>n]}\right| \rightarrow 0$ as $n \rightarrow \infty$. Assume

$$
t P^{*}(F>t) \rightarrow 0 \quad \text { as } t \rightarrow \infty
$$


and condition (iii) from Theorem 5.4. Then

$$
\left\|\sum_{i=1}^{n}\left(f\left(X_{i}\right)-P f\right) / n\right\|_{\mathscr{F}} \rightarrow 0 \text { in } \mathrm{pr}^{*} .
$$

If instead of (5.10) we have $P^{*} F<\infty$, then

$$
\left\|\sum_{i=1}^{n}\left(f\left(X_{i}\right)-P f\right) / n\right\|_{\mathscr{F}} \rightarrow 0 \quad \text { a.s. }
$$

(It is easy to check that the condition $\sup _{f \in \mathscr{F}}\left|P f I_{[|f|>n]}\right| \rightarrow 0$ allows for centering at expectations.)

5.9. EXAMPLE. A stable CLT in $c_{0}$. The positive results in the following proposition are obtained as corollaries to the theorems in this section. Recall that $c_{0}$ denotes the Banach space of all real sequences that converge to zero, equipped with the sup norm. If $X$ is a $c_{0}$-valued random variable, we write $X \in \operatorname{CLT}\left(n^{1 / p}\right)$, $p \in(1,2]$, if there is a $p$-stable measure $\gamma$ in $c_{0}$ such that

$$
\mathscr{L}\left(\sum_{i=1}^{n}\left(X_{i}-E X_{i}\right) / n^{1 / p}\right) \underset{w}{\rightarrow} \gamma
$$

where the $X_{i}$ are i.i.d. copies of $X$. If $\gamma=\delta_{0}$ we write $X \in \operatorname{LLN}\left(n^{1 / p}\right)$ and if the sequence $\left\{\left\|\sum_{i=1}^{n}\left(X_{i}-E X_{i}\right) / n^{1 / p}\right\|\right\}_{n=1}^{\infty}$ is stochastically bounded, $X \in \operatorname{BCLT}\left(n^{1 / p}\right)$. With this notation we have

5.10. PROPOSITION. Let $X=\left(\varsigma_{1}, \ldots, \varsigma_{k}, \ldots\right)$ be a random vector with infinitely many real coordinates. Let $p \in(1,2)$ and let $p^{\prime}$ be the conjugate of $p$. Set $\varepsilon_{n}:=(L n)^{1 / p^{\prime}} \Lambda_{p, \infty}\left(\sup _{j \geq n}\left|\zeta_{j}\right|\right)$. We have:

(i) If the sequence $\left\{\varepsilon_{n}\right\}$ is bounded, then $X \in c_{0}$ and $X \in \operatorname{BCLT}\left(n^{1 / p}\right)\left(\right.$ in $\left.c_{0}\right)$.

(ii) If $\left(\varsigma_{1}, \ldots, \varsigma_{k}\right) \in \operatorname{CLT}\left(n^{1 / p}\right)$ for all $k \in \mathbf{N}$ and if $\lim _{n \rightarrow \infty} \varepsilon_{n}=0$, then $X \in \operatorname{CLT}\left(n^{1 / p}\right)$.

(iii) If $\lim _{t \rightarrow \infty} t^{p} P\left\{\left|\zeta_{k}\right|>t\right\}=0$ and $\lim _{n \rightarrow \infty} \varepsilon_{n}=0$, then $X \in \operatorname{LLN}\left(n^{1 / p}\right)$. Moreover, the $c_{0}$-valued random variable $Y=\left\{(L k)^{-1 / p^{\prime}} \varepsilon_{k}\right\}_{k=1}^{\infty}$, where $\left\{\varepsilon_{k}\right\}$ is a Rademacher sequence, does not belong to $\operatorname{LLN}\left(n^{1 / p}\right)$, and $Z=\left\{c_{k}(L k)^{-1 / p^{\prime}} \varepsilon_{k}\right\}_{k=1}^{\infty}$, where $c_{k} \rightarrow \infty$, does not belong to $\operatorname{BCLT}\left(n^{1 / p}\right)$.

The statements about $Y$ and $Z$ follow directly from Kolmogorov's lower exponential bound (e.g. Stout (1974, p. 262, Theorem 5.2.2(iii))) as in the proof of Proposition 5.4 in Andersen et al. (1988). Details are omitted. Statement (iii) is a particular case of (ii) (with $\delta_{0}$ as limiting measure), and the proof of (i) is similar to the proof of (ii), so we will only prove (ii). The proof is based on Theorem 5.2 and the following lemma, which explicitly construct majorizing measures for some simple ultrametric spaces (this lemma is known; it is only presented here for the readers' convenience). See Heinkel (1983) for a similar construction. See also Paulauskas, and Rackauskas (1984) for results similar to (in fact contained in) Proposition 5.10.

5.11. LeMMA. Let $1<p \leq 2$ and let $p^{\prime}$ denote the conjugate of $p$. Let $\overline{\mathbf{N}}=\mathbf{N} \cup\{\infty\}$, let $\left\{a_{n}: n \in \mathbf{N}\right\}$ be a sequence of positive numbers and set $a_{\infty}=0$. 
Define on $\overline{\mathbf{N}}$ a distance $\rho$ by

$$
\rho(i, j)=a_{i} \vee a_{j}, \quad i, j \in \overline{\mathbf{N}}, i \neq j ; \quad \rho(i, i)=0, \quad i \in \overline{\mathbf{N}} .
$$

Then, if $a_{n}=o\left((L n)^{-1 / p^{\prime}}\right)$ there are projections $\pi_{q}: \overline{\mathbf{N}} \rightarrow \overline{\mathbf{N}}, q \in \mathbf{N}$, and $a$ probability measure $\mu$ on $\bigcup_{q=1}^{\infty} \pi_{q} \overline{\mathbf{N}}$ satisfying:

(a) $\pi_{q}$ has finite range and $\rho\left(j, \pi_{q} j\right) \leq 2^{-q}, j \in \overline{\mathbf{N}}, q \in \mathbf{N}$, and

(b) $\lim _{q_{0} \rightarrow \infty} \sup _{j \in \overline{\mathrm{N}}} \sum_{q \geq q_{0}} 2^{-q}\left(\ln 1 / \mu\left\{\pi_{q} j\right\}\right)^{1 / p^{\prime}}=0$ with the $\sup _{j}$ finite for $q_{0}=1$. If $a_{n}=O\left((L n)^{-1 / p^{\prime}}\right)$ then there are $\pi_{q}, q \in \mathbf{N}$, and $\mu$ satisfying (a) and

$\left(\mathrm{b}^{\prime}\right) \sup _{j} \sum_{q \geq 1} 2^{-q}\left(\ln 1 / \mu\left\{\pi_{q} j\right\}\right)^{1 / p^{\prime}}<\infty$.

ProOF. Only the "little $o$ " statement will be proved (the "big $O$ " part is analogous). Obviously if $(\overline{\mathbf{N}}, \bar{\rho})$ satisfies (a) and (b), so does $(\overline{\mathbf{N}}, \rho)$ for any $\rho$ such that $\rho \leq c \bar{\rho}, c<\infty$. Hence we may assume $a_{1}=1, a_{n}=\varepsilon_{n}(L n)^{-1 / p^{\prime}}$ with $a_{n} \downarrow 0$, $\varepsilon_{n} \downarrow 0$ and $\varepsilon_{n} \geq(L n)^{-1}$. Let

$$
n_{0}:=0 \text { and } n_{q}:=\inf \left\{j: a_{j} \leq 2^{-q}\right\} \text { for } q \geq 1,
$$

and define

$$
\pi_{q} j=j \quad \text { if } j<n_{q}, \quad \pi_{q} j=\infty \quad \text { otherwise. }
$$

Then obviously $\pi_{q}$ satisfies (a). Define a probability measure $\mu$ on $\overline{\mathbf{N}}$ as follows:

$$
\mu\{\infty\}=\frac{1}{2}, \quad \mu\{j\}=c / n_{q}^{2} \text { for } n_{q-1}<j \leq n_{q}, q \in \mathbf{N},
$$

where $c=\left[2 \sum_{q=1}^{\infty}\left(n_{q}-n_{q-1}\right) / n_{q}^{2}\right]^{-1}$. (Note that since $\varepsilon_{n}>(L n)^{-1}, \sum n_{q}^{-1}<\infty$.) Now we check that $\mu$ satisfies condition (b). The sequence $n_{q}$ is monotonically increasing to $\infty$ and, since $\varepsilon_{n} \downarrow 0$, there exist $\delta_{q} \rightarrow 0$ such that

$$
n_{q}=\exp \left\{2^{q p^{\prime}} \delta_{q}\right\}, \quad q \in \mathbf{N} .
$$

If $j<n_{q_{0}}$ then $\pi_{q} j=j$ for all $q \geq q_{0}$, and $\mu\{j\} \geq \mu\left\{n_{q_{0}}\right\}$. Hence

$$
\begin{aligned}
\sum_{q=q_{0}}^{\infty} 2^{-q}\left(\ln \frac{1}{\mu\left\{\pi_{q} j\right\}}\right)^{1 / p^{\prime}} & \leq\left(\sum_{q=q_{0}}^{\infty} 2^{-q}\right)\left(|\ln c|+2^{1+q_{0} p^{\prime}} \delta_{q_{0}}\right)^{1 / p^{\prime}} \\
& =\left(2^{\left(1-q_{0}\right) p^{\prime}}|\ln c|+2^{p^{\prime}+1} \delta_{q_{0}}\right)^{1 / p^{\prime}} .
\end{aligned}
$$

If $j \geq n_{q_{0}}$ and if $q_{j}, j>0$, is defined by the inequalities $n_{q_{j-1}} \leq j<n_{q_{j}}$, we have (since $\pi_{q} j=\infty$ for $q<q_{j}$, and $\mu\left\{\pi_{q} j\right\}=\mu\{j\} \geq \mu\left\{n_{q_{j}}\right\}$ for $q \geq q_{j}$ ):

$$
\begin{aligned}
\sum_{q=q_{0}}^{\infty} & 2^{-q}\left(\ln \frac{1}{\mu\left\{\pi_{q} j\right\}}\right)^{1 / p^{\prime}} \\
& =\left(\sum_{q=q_{0}}^{q_{j}-1} 2^{-q}\right)(\ln 2)^{1 / p^{\prime}}+\left(\sum_{q=q_{j}}^{\infty} 2^{-q}\right)\left(|\ln c|+2^{1+q_{j} p^{\prime}} \delta_{q_{j}}\right)^{1 / p^{\prime}} \\
& \leq 2^{-q_{0}+1}(\ln 2)^{1 / p^{\prime}}+\left(2^{\left(1-q_{j}\right) p^{\prime}}|\ln c|+2^{p^{\prime}+1} \delta_{q_{j}}\right)^{1 / p^{\prime}}
\end{aligned}
$$

(5.15) and (5.16) prove (b).

PROOF OF (ii) FROM PROPOSITION 5.10. Let $(S, \mathscr{S})=\left(c_{0}, \mathscr{B}\right), P=$ $\mathscr{L}\left(\xi_{1}, \ldots, \xi_{n}, \ldots\right)=\mathscr{L}(X)$ and $\mathscr{F}=\left\{f_{n}, n \in \bar{N}: f_{n}(x)=x_{n}\right.$ for $n<\infty$, $\left.f_{\infty}(x)=0\right\}$. It is enough to prove that $\mathscr{F} \in \operatorname{CLT}\left(P, n^{1 / p}\right)$. We apply Theo- 
rem 5.2. The finite dimensional distributions satisfy the $n^{1 / p}$-CLT by hypothesis. Let $a_{n}=\varepsilon_{n} /(L n)^{1 / p^{\prime}}, n \in \mathbf{N}$, and $a_{\infty}=0$, and define

$$
\rho\left(f_{n}, f_{m}\right)=a_{n \wedge m} \quad \text { if } n \neq m, \rho\left(f_{n}, f_{n}\right)=0, n, m \in \overline{\mathbf{N}} .
$$

Then, by Lemma $5.11,(\mathscr{F}, \rho)$ satisfies condition (iiia) from Theorem 5.2. To verify conditions (ii) and (iiib) from Theorem 5.2 with $B(n)=n^{1 / p}$ note that, given $\varepsilon>0$, if $a_{n} \leq \varepsilon<a_{n-1}$, then $B_{\rho}\left(f_{k}, \varepsilon\right)=\left\{f_{k}\right\}$ if $k<n$ and $B_{\rho}\left(f_{k}, \varepsilon\right) \subseteq\left\{f_{j}\right\}_{j \geq n}$ for $k \geq n$; therefore,

$$
\begin{aligned}
& \sup _{t>0} t \operatorname{Pr}\left\{\sup _{\rho(f, g) \leq \varepsilon}|f(X)-g(X)|>\varepsilon t^{1 / p}\right\} \\
& \leq 2^{1+p} \varepsilon^{-p} \sup _{t>0} t^{p} \operatorname{Pr}\left\{\sup _{j \geq n}\left|\xi_{j}\right|>t\right\} \leq 2^{1+p}
\end{aligned}
$$

and this gives (ii) and (iiib) by Remark 5.3(2).

6. A law of the iterated logarithm. In Andersen et al. (1988) and in Ledoux and Talagrand (1986) the law of the iterated logarithm of Ledoux (1981) is extended to a bracketing LIL. Here we extend the following recent result of Ledoux (private communication): Let $(T, d)$ be a compact metric space, $\rho$ a $d$-continuous pseudodistance on $T, M$ a real random variable and $X(t), t \in T$, a sample continuous process such that:

(i) $E X^{2}(t)<\infty$ for all $t \in T$ (respectively, the random variables $\left\{X^{2}(t): t \in T\right\}$ are uniformly integrable),

(ii) $|X(t, \omega)-X(s, \omega)| \leq M(\omega) \rho(s, t)$ for all $\omega \in \Omega, s, t \in T$,

(iii) $\rho$ is dominated by the $L_{2}$-distance of a sample bounded Gaussian process (respectively, sample continuous Gaussian process),

(iv) $E\left(M^{2} / L_{2} M\right)<\infty$.

Then $X$ satisfies the bounded law of the iterated logarithm (BLIL) (respectively, the compact law of the iterated logarithm-the CLIL).

A consequence of our result is that the condition on $M$ can be weakened to $\sup _{t>0}\left(t^{2} / L_{2} t\right) P(M>t)<\infty$.

(As usual, $L x=1 \vee \ln x$ and $L_{2}=L \circ L$. We refer to Andersen et al. (1988) for notation on the LIL for empirical processes, in particular for the meaning of expressions like $\mathscr{F} \in \operatorname{CLIL}(P)$ and $\mathscr{F} \in \operatorname{BLIL}(P)$.)

The additional ingredient for the proof of the following LIL will be the LedouxTalagrand (1986) LIL for $B$-valued random variables $X, B$ a separable Banach space.

6.1. TheOREM (LedouX AND TAlagrand (1986)). Let $X$ be a B-valued random variable, $B$ a separable Banach space. Then $X \in \mathrm{CLIL}$ (resp. $X \in \mathrm{BLIL}$ ) if and only if the following three conditions hold:

(i) $E f(X)=0$ and the random variables $\left\{f^{2}(X): f \in B^{\prime},\|f\|<1\right\}$ are uniformly integrable (resp. $E f(X)=0$ and $E f^{2}(X)<\infty$ for all $f \in B^{\prime}$ ).

(ii) $E\left(\|X\|^{2} / L_{2}\|X\|\right)<\infty$.

(iii) If $\left\{X_{i}\right\}$ are i.i.d. copies of $X$ and $\left\{\varepsilon_{i}\right\}$ a Rademacher sequence independent of $\left\{X_{i}\right\}$, then $\sum_{i=1}^{n} \varepsilon_{i} X_{i} / a_{n} \rightarrow 0$ in pr. (resp. the sequence $\left\{\left\|\sum_{i=1}^{n} \varepsilon_{i} X_{i} / a_{n}\right\|\right\}_{n=1}^{\infty}$ is stochastically bounded) where $a_{n}=\left(n L_{2} n\right)^{1 / 2}$. 
Actually, the proof of this theorem goes through in the nonseparable case if enough measurability is assumed. So, Theorem 5.1 reduces the LIL to a weak law of large numbers, which is essentially the type of result we ended up proving in the previous sections.

6.2. THEOREM. Let $\mathscr{F} \subset \mathscr{L}_{2}(S, \mathscr{S}, P)$ with envelope $F$ everywhere finite, and such that for all $n \in \mathbf{N}$, the processes

$$
f \rightarrow \sum_{i=1}^{n} f\left(X_{i}\right), \quad f \in \mathscr{F},
$$

are separable $\left(\right.$ for $\left.\mathscr{L}_{1}(P)\right)$. Assume:

$$
P\left(F^{2} / L_{2} F\right)<\infty,
$$

and that there exists a Gaussian process $G$ on $\mathscr{F}$ with bounded $d_{G}$-uniformly continuous sample paths $\left(d_{G}^{2}(f, g)=E(G(f)-G(g))^{2}\right)$ (respectively, $G$ has bounded sample paths) such that

$$
\sup _{f, g \in \mathscr{G}} P\left\{\frac{(f-g)^{2} / d_{G}^{2}(f, g)}{L_{2}\left[|f-g| / d_{G}(f, g)\right]}\right\}<\infty
$$

and

$$
\text { there exists } K<\infty \text { such that for all } f \in \mathscr{F}, \varepsilon>0 \text {, }
$$

$$
\sup _{t>0} \frac{t^{2}}{L_{2} t} \operatorname{Pr}\left\{\varepsilon^{-1} \sup _{g \in B_{d_{G}}(f, \varepsilon)}|f-g|>t\right\} \leq K .
$$

Then $\mathscr{F} \in \operatorname{CLIL}(P)$ (respectively, $\mathscr{F} \in \operatorname{BLIL}(P))$.

PrOOF. We will only prove the compact LIL. Since $\{G(f): f \in \mathscr{F}\}$ is relatively compact in $\mathscr{L}_{2}(S, \mathscr{S}, P)$, the family of functions $\left\{\left(\sum \alpha_{i} G\left(f_{i}\right)\right)^{2}: f_{i} \in \mathscr{F}, \sum\left|\alpha_{i}\right| \leq\right.$ $1\}$ is uniformly integrable by Dini's lemma. So, by Theorem 6.1 , we only need to show that for all $\varepsilon>0$,

$$
\lim _{n} \operatorname{Pr}\left\{\left\|\sum_{i=1}^{n} \varepsilon_{i} f\left(X_{i}\right) / a_{n}\right\|_{\mathscr{G}}>\varepsilon\right\}=0 .
$$

Now, by Talagrand's (1987) theorem on Gaussian processes, if $\varphi(x)=x^{1 / 2}$ then there is a discrete probability measure $\mu$ on $\mathscr{F}$ supported by $\left\{\pi_{q} f: q \in \mathbf{N}, f \in \mathscr{F}\right\}$ such that (4.7) holds with $\rho=d_{G}$, and therefore (4.9) holds with $\varphi^{-1}(x)=x^{2}$. Define

$$
c_{n, q}(f)=2^{-q}\left(n L_{2} n\right)^{1 / 2} / \gamma_{q} f
$$

and then $\tau f$ and $\Delta_{q} f$ as in the proof of Theorem 4.2 using this new $c_{n, q} f$. Since for every $q_{0}, \#\left\{\pi_{q_{0}} f: f \in \mathscr{F}\right\}<\infty$ and $\pi_{q_{0}} f \in \mathscr{L}_{2}(P)$,

$$
\left\|\sum_{i=1}^{n} \varepsilon_{i}\left(\pi_{q_{0}} f\right)\left(X_{i}\right) / a_{n}\right\|_{\mathscr{F}} \rightarrow 0 \text { in pr. }
$$


hence (6.1) is equivalent to

$$
\lim _{q_{0} \rightarrow \infty} \limsup _{n} \operatorname{Pr}\left\{\left\|\sum_{i=1}^{n} \varepsilon_{i}\left(f-\pi_{q_{0}} f\right)\left(X_{i}\right) / a_{n}\right\|_{\mathscr{F}}>\varepsilon\right\}=0
$$

for all $\varepsilon>0$. To prove (6.2) we use a decomposition of $f-\pi_{q_{0}} f$ just as in (4.14), that is

$$
\begin{aligned}
\operatorname{Pr}\left\{\left\|\sum_{i=1}^{n} \varepsilon_{i}\left(f-\pi_{q_{0}} f\right)\left(X_{i}\right) / a_{n}\right\|_{\mathscr{F}}>4 \varepsilon\right\} \\
\leq \operatorname{Pr}\left\{\left\|\sum_{i=1}^{n} \varepsilon_{i}\left(f-\pi_{q_{0}} f\right) I_{\left[\tau f=q_{0}\right]}\left(X_{i}\right) / a_{n}\right\|_{\mathscr{F}}>\varepsilon\right\} \\
+\operatorname{Pr}\left\{\left\|\sum_{i=1}^{n} \varepsilon_{i}\left(f-\pi_{q_{1}} f\right) I_{\left[\tau f \geq q_{1}\right]}\left(X_{i}\right) / a_{n}\right\|_{\mathscr{F}}>\varepsilon\right\} \\
+\operatorname{Pr}\left\{\left\|\sum_{q=q_{0}+1}^{q_{1}-1} \sum_{i=1}^{n} \Delta_{q} f I_{\left[c_{n, q+1} f<\Delta_{q} f \leq c_{n, q} f\right]}\left(X_{i}\right) / a_{n}\right\|_{\mathscr{F}}>\varepsilon\right\} \\
+\operatorname{Pr}\left\{\left\|\sum_{q=q_{0}+1}^{q_{1}} \sum_{i=1}^{n} \varepsilon_{i}\left(\pi_{q} f-\pi_{q-1} f\right) I_{[\tau f \geq q]}\left(X_{i}\right) / a_{n}\right\|_{\mathscr{F}} \geq \varepsilon\right\} \\
:=(\mathrm{II})+(\mathrm{III})+(\mathrm{IV})+(\mathrm{V}) .
\end{aligned}
$$

The integrability condition (i) implies that

$$
n P\left\{F>\lambda a_{n}\right\} \rightarrow 0 \text { as } n \rightarrow \infty
$$

for all $\lambda \rightarrow 0$. So, assuming as we may that $\gamma_{q_{0}+1} f \leq 2^{q_{0}}$, we have

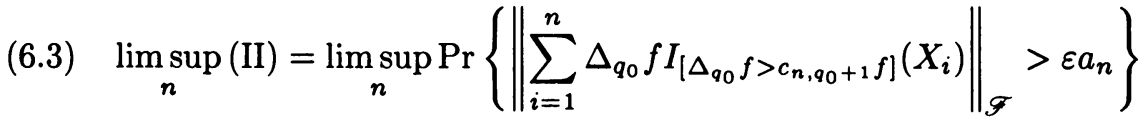
$\leq \underset{n}{\lim \sup } n P\left\{2 F>2^{-2 q_{0}} a_{n}\right\}=0$.

As in Theorem 4.2,

$$
\text { (III) }=0
$$

if $n c_{n, q_{1}+1} f \leq \varepsilon a_{n}$ or, if $n 2^{-q_{1}} \leq \varepsilon \gamma_{q_{1}+1} f$, and since $\gamma_{q_{1}+1} f \geq\left(q_{1}+1\right)^{1 / 2}$, this holds for $\ln n>\varepsilon^{-2}$ as long as $q_{1}(n) \geq L n$. For (IV), we use the proof of Theorem 
3.1 with $h_{l}=l^{-1 / 2}, \psi(x) \simeq x$ and $d=1$. We get, as in the estimation of (I) in Theorem 3.1,

$$
\begin{aligned}
& (\mathrm{V}) \leq \sum_{q=q_{0}+1}^{q_{1}-1} \sum_{t \in T_{q}} \operatorname{Pr}\left\{\sup _{\left\{f: \pi_{q} f=t\right\}} \sum_{i=1}^{n} \Delta_{q} f I_{[\tau=q]}\left(X_{i}\right)>\varepsilon \beta_{q_{0}+2}^{-1} a_{n} 2^{-q-1} \gamma_{q+1} f\right\} \\
& =\sum_{q=q_{0}+1}^{q_{1}-1} \sum_{t \in T_{q}} \operatorname{Pr}\left\{\sup _{\left\{f: \pi_{q} f=t\right\}} \frac{2^{-1}}{\gamma_{q+1} f}\right.
\end{aligned}
$$

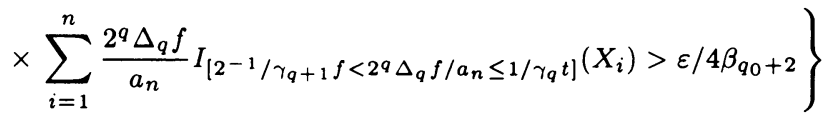

$$
\begin{aligned}
& \leq \sum_{q=q_{0}+1}^{q_{1}-1} \sum_{t \in T_{q}} \sum_{c b_{t}^{-2}<l \leq n}\left[\frac{\left.e n P\left\{2^{q} \Delta_{q} f / a_{n}>c / l\right)^{1 / 2}\right\}}{l}\right]^{l} \\
& \text { (where we let } b_{t}:=1 / \gamma_{q} t \text { and } c:=\varepsilon / 4 \beta_{q_{0}+2} \text { ) } \\
& \leq \sum_{q=q_{0}+1}^{q_{1}-1} \sum_{t \in T_{q}} \sum_{c b_{t}^{-2}<l \leq n}\left[\frac{e n}{l} \frac{K L_{2}\left(\left(c n L_{2} n / l\right)^{1 / 2}\right)}{c n L_{2} n / l}\right]^{l}
\end{aligned}
$$

Now, for $L_{2} c>2$ and $L_{2} n>2$ (which we can assume), we have

$$
L_{2}\left(\left(\frac{c n L_{2} n}{l}\right)^{1 / 2}\right) \leq L_{2}(c n)=L(L c+L n) \leq L_{2} c+L_{2} n \leq\left(L_{2} c\right)\left(L_{2} n\right)
$$

So, by (4.9) with $\varphi^{-1}(x)=x^{2}$,

$$
\begin{aligned}
(\mathrm{V}) & \leq \sum_{q=q_{0}+1}^{q_{1}-1} \sum_{t \in T_{q}} \sum_{c b_{t}^{-2}<l \leq n}\left[\frac{K e L_{2} c}{c}\right]^{l} \\
& \leq \sum_{q=q_{0}+1}^{q_{1}-1} \sum_{t \in T_{q}}\left(1-\frac{K e L_{2} c}{c}\right)^{-1} \exp \left\{-c b_{t}^{-2} \ln \left(c / K e L_{2} c\right)\right\} \\
& \rightarrow 0 \text { as } q_{0} \rightarrow \infty
\end{aligned}
$$

Finally, to estimate (V) we must find a bound for $P\left(\pi_{q} f-\pi_{q-1} f\right)^{2} I_{\left[\tau_{j} \geq q\right]}$. Noting that $d_{G}^{2}\left(\pi_{q} f, \pi_{q-1} f\right) \leq 9 \cdot 2^{-2 q}$, that $c_{n, q} f / 3 \cdot 2^{-q} \leq n$, and using hypothesis (ii) we get

$$
\begin{aligned}
P\left(\pi_{q} f-\pi_{q-1} f\right)^{2} I_{\left[\tau_{j} \geq q\right]} & \leq K d_{G}^{2}\left(\pi_{q} f, \pi_{q-1} f\right) L_{2}\left(\frac{c_{n, q} f}{d_{G}\left(\pi_{q} f, \pi_{q-1} f\right)}\right) \\
& \leq 9 K 2^{-2 q} L_{2} n
\end{aligned}
$$


Hence, using Bernstein's inequality as in the evaluation of (V) in Theorem 4.2, we get

$$
\begin{aligned}
&(\mathrm{V}) \leq 2 \sum_{q=q_{0}+1}^{q_{1}} \sum_{t \in T_{q}} \operatorname{Pr}\left\{\left|\sum_{i=1}^{n} \varepsilon_{i}\left(\pi_{q} t-\pi_{q-1} t\right) I_{[\tau \leq q]}\left(X_{i}\right)\right|>\varepsilon a_{n} 2^{-q} \gamma_{q} t / \beta_{q_{0}+1}\right\} \\
& \leq 2 \sum_{q=q_{0}+1}^{q_{1}} \sum_{t \in T_{q}} \exp \left\{\frac{-\varepsilon^{2} a_{n}^{2} 2^{-2 q}\left(\gamma_{q} t\right)^{2} / \beta_{q_{0}+1}^{2}}{18 K 2^{-2 q} a_{n}^{2}+\frac{\varepsilon}{2 \beta_{q_{0}+2}} a_{n} 2^{-q}\left(\gamma_{q} t\right)\left(c_{n, q} t\right)}\right\} \\
& \leq 2 \sum_{q=q_{0}+1}^{q_{1}} \sum_{t \in T_{q}} \exp \left\{-\frac{\left(\gamma_{q} t\right)^{2}}{\beta_{q_{0}+1}^{2}\left(18 K+3 \varepsilon / 2 \beta_{q_{0}+1}\right)}\right\} \rightarrow 0 \\
& \text { as } q_{0} \rightarrow \infty \text { uniformly in } n .
\end{aligned}
$$

(6.3) to (6.5) give (6.2) and the result follows.

For another approach to the LIL (and the CLT $\left(n^{1 / 2}\right)$ ) for Lipschitz processes see Heinkel (1987).

7. Some additional examples: $\mathrm{BL}^{*}$ norms, weighted empiricals.

(a) $\mathrm{BL}^{*}$ norms. For $f: \mathbf{R} \rightarrow \mathbf{R}$, define

$$
\begin{gathered}
\|f\|_{\mathrm{BL}}:=\|f\|_{\infty}+\sup _{x \neq y}|f(x)-f(y)| /|x-y| \\
\mathrm{BL}_{1}(\mathbf{R}):=\left\{f: \mathbf{R} \rightarrow \mathbf{R},\|f\|_{\mathrm{BL}} \leq 1\right\}
\end{gathered}
$$

and for Borel measures $Q$ of bounded variation on $\mathbf{R}$,

$$
\|Q\|_{\mathrm{BL}^{*}}=\sup \left\{\left|\int f d Q\right|: f \in \mathrm{BL}_{1}(\mathbf{R})\right\} .
$$

It is well known that $\left\|P_{n}-P\right\|_{\mathrm{BL}^{*}} \rightarrow 0$ a.s. for all probability laws $P$ on $(\mathbf{R}, \mathscr{B})$, and Giné and Zinn (1986) prove that $\operatorname{BL}_{1}(\mathbf{R}) \in \operatorname{CLT}\left(P, n^{1 / 2}\right)$ if and only if $\sum_{j=-\infty}^{\infty}\left[P\left(I_{j}\right)\right]^{1 / 2}<\infty$, where $I_{j}=[j-1, j)$. A natural question to ask is whether $\mathrm{BL}_{1}(\mathbf{R}) \in \operatorname{CLT}\left(P, n^{1 / p}\right), p \in(1,2)$, under weaker conditions on $P$. A first observation is that $\mathrm{BL}_{1}(\mathbf{R}) \in \operatorname{CLT}\left(P, n^{1 / p}\right)$ if and only if

$$
n^{1-1 / p}\left\|P_{n}-P\right\|_{\mathrm{BL}^{*}} \rightarrow 0 \text { a.s. }
$$

because of boundedness of the envelope function $F$ of $\mathrm{BL}_{1}(\mathbf{R})$. We obtain below necessary and sufficient conditions for (7.1) to hold. We do not seem to be able to obtain this result in full from Proposition 5.7, but it gets close to it. As an illustration, here is how one applies this proposition. To apply Proposition 5.7 for a given $P$, it is enough to find a distance $\rho$ satisfying conditions (iii) from Theorem 5.2 with $B(t)=t^{1 / p}$ and $\mathscr{F}=\mathrm{BL}_{1}(\mathbf{R})$. For clarity of exposition, we will assume $p_{j}:=P\left(I_{j}\right) \downarrow$ and $p_{j}=0$ for $j \leq 0$, and will only be interested in $p_{j}$ of power type. Consider, for $f, g \in \mathrm{BL}_{1}\left(\mathbf{R}^{+}\right)$,

$$
\rho(f, g)=\sup _{j \in \mathbf{N}}\|f-g\|_{j} / j^{\tau}, \quad \text { with }\|h\|_{j}=\sup \left\{|h(x)|: x \in I_{j}\right\}
$$


where $\tau$ is a positive number to be determined. Standard computations show that the metric entropy of $\mathrm{BL}_{1}\left(\mathbf{R}^{+}\right)$for $\rho$ is

$$
\ln N\left(\mathrm{BL}_{1}\left(\mathbf{R}^{+}\right), \rho, \varepsilon\right) \simeq \varepsilon^{-1} \sum_{j \leq \varepsilon^{-1 / \tau}} j^{-\tau} \simeq\left\{\begin{array}{l}
\varepsilon^{-1 / \tau} \text { if } \tau<1, \\
\varepsilon^{-1} \ln \varepsilon^{-1} \text { if } \tau=1, \\
\varepsilon^{-1} \text { if } \tau>1,
\end{array}\right.
$$

and we want $\rho$ to satisfy (iiia) in Theorem 5.2 , which for metric entropy reduces to

$$
\int_{0}\left(\ln N\left(\mathrm{BL}_{1}\left(\mathbf{R}^{+}\right), \rho, \varepsilon\right)\right)^{1 / p^{\prime}} d \varepsilon<\infty
$$

(with $1 / p+1 / p^{\prime}=1$ ). This integral is finite if and only if $\tau>1 / p^{\prime}$.

Condition (iiib) in Theorem 5.2 for $P$ becomes

$$
\sup _{t>0} t^{p} P\left\{x: \sup \left[|h(x)|: h \in \mathrm{BL}_{1}\left(\mathbf{R}^{+}\right),\|h\|_{j} \leq \varepsilon j^{\tau} \text { for } j<\varepsilon^{-1 / \tau}\right]>t\right\}<c \varepsilon^{p}
$$

for all $\varepsilon$ near to 0 . The left-hand side is bounded by

$$
\sup _{t>0} t^{p}\left[\sum_{j<\varepsilon^{-1 / \tau}} p_{j} I_{\left[\varepsilon j^{\tau}>t\right]}+\sum_{j \geq \varepsilon^{-1 / \tau}} p_{j} I_{[1>t]}\right],
$$

and this is bounded by $\varepsilon^{p} c$ for all $\varepsilon$ near to 0 if and only if $\sup _{t>0} t^{\tau_{p}} \sum_{j>t} p_{j}<\infty$. Thus, Proposition 5.7 shows that if $p_{j} \leq 1 / j^{r}$ for some $r>p$, then (7.1) holds.

Now we will obtain general necessary and sufficient conditions for (7.1) to hold; in particular we will see that if $p_{j}=1 / j^{p}$ then $P$ does not satisfy (7.1). Note that with extra care in the above arguments we would have obtained (7.1) for $p_{j}=1 / j^{p}(\ln j)^{r}$ for certain $r$ 's, but not much more, and this is not good enough as we see below.

7.1. TheOREM. Let $p_{j}=P\left(I_{j}\right), j \in \mathbf{Z}$. Then

$$
n^{1-1 / p}\left\|P_{n}-P\right\|_{\mathrm{BL}} \rightarrow 0 \quad \text { a.s. }
$$

if and only if both

$$
n^{1-1 / p} \sum_{\left\{j: p_{j} \leq 1 / n\right\}} p_{j} \rightarrow 0
$$

and

$$
n^{1 / 2-1 / p} \sum_{\left\{j: p_{j}>1 / n\right\}} p_{j}^{1 / 2} \rightarrow 0 .
$$

ProOF. We decompose $f(x), f \in \mathrm{BL}_{1}(\mathbf{R})$, as follows,

$$
\begin{aligned}
f(x)= & \sum_{j=-\infty}^{\infty} I_{[x \in[j-1, j)]}\left[\int_{j-1}^{x}\left(f^{\prime}(s)-f(j)+f(j-1)\right) d s\right. \\
& \quad+(f(j)-f(j-1))(x-j+1)+f(j-1)] \\
= & \sum_{j=-\infty}^{\infty} \int_{j-1}^{j} I_{[x \in[j-1, j)]} I_{[j-1, x]}(s) \varphi_{j}(s) d s \\
& +\sum_{j=-\infty}^{\infty} a_{j}\left[(x-j+1) I_{[x \in[j-1, j)]}+(j+1-x) I_{[x \in[j, j+1)]}\right],
\end{aligned}
$$


where $f^{\prime}$ is the derivative of $f, \varphi_{j}(s)=f^{\prime}(s)-(f(j)-f(j-1))$ and $a_{j}=f(j)$. Let

$$
T: \mathrm{BL}(\mathbf{R}) \rightarrow \mathbf{L}^{\infty}:=\left[\bigoplus_{l_{\infty}}^{\infty}{ }_{j=-\infty}^{\infty} L_{0}^{\infty}(j-1, j)\right] \oplus_{l^{\infty}} l^{\infty}(\mathbf{Z})
$$

where

$$
L_{0}^{\infty}(j-1, j):=\left\{\varphi \in L^{\infty}(j-1, j): \int_{j-1}^{j} \varphi(s) d s=0\right\},
$$

be defined by $T f=\left\{\left\{\varphi_{j}\right\}_{j=1}^{\infty},\left\{a_{j}\right\}_{j=1}^{\infty}\right\}$ with $\varphi_{j}, a_{j}$ as above. Then $T$ is obviously an isomorphism between these two Banach spaces.

By randomization, as usual, we can replace in (7.1) $\left(P_{n}-P\right)(f)$ by $\sum_{i=1}^{n} \varepsilon_{i} f\left(X_{i}\right)$, $X_{i}$ i.i.d. with law $P$. The decomposition (7.4) gives, by duality (note that $\left(\mathrm{L}^{\infty}\right)^{\prime}=$ $\left.\mathbf{L}^{1}:=\left[\bigoplus_{l^{1}}\left(L^{1}(j-1, j) / \mathbf{R}\right)\right] \oplus_{l^{1}} l^{1}(\mathbf{Z})\right)$,

$$
\begin{aligned}
& \left\|\sum_{i=1}^{n} \varepsilon_{j} f\left(X_{j}\right)\right\|_{\mathrm{BL}_{1}(\mathbf{R})} \\
& \simeq \sum_{j=-\infty}^{\infty} \inf _{a \in \mathbf{R}} \int_{j-1}^{j}\left|\sum_{i=1}^{n} \varepsilon_{i} I_{\left[j-1, X_{i}\right)}(s) I_{\left[X_{i} \in[j-1, j)\right]}+a\right| d s \\
& \quad+\sum_{j=-\infty}^{\infty}\left(\left|\sum_{i=1}^{n} \varepsilon_{i}\left(X_{i}-j+1\right) I_{\left[X_{i} \in[j-1, j)\right]}\right|\right. \\
& \left.\quad+\left|\sum_{i=1}^{n} \varepsilon_{i}\left(j+1-X_{i}\right) I_{\left[X_{i} \in[j, j+1)\right]}\right|\right) .
\end{aligned}
$$

So, by the contraction principle,

$$
E_{\varepsilon}\left\|\sum_{i=1}^{n} \varepsilon_{i} f\left(X_{i}\right)\right\| \|_{\mathrm{BL}_{1}(\mathbf{R})} \lesssim \sum_{j=-\infty}^{\infty} E_{\varepsilon}\left|\sum_{i=1}^{n} \varepsilon_{i} I_{\left[X_{i} \in[j-1, j)\right]}\right|,
$$

and by the triangle inequality (applied to the last two summands in (7.5) with a shift on $j$ for the third),

$$
\left\|\sum_{i=1}^{n} \varepsilon_{i} f\left(X_{i}\right)\right\|_{\mathrm{BL}_{1}(\mathbf{R})} \gtrsim \sum_{j=-\infty}^{\infty}\left|\sum_{i=1}^{n} \varepsilon_{i} I_{\left[X_{i} \in[j-1, j)\right]}\right| .
$$

Now, $E\left|\sum_{i=1}^{n} \varepsilon_{i} I_{\left[X_{i} \in[j-1, j]\right.}\right|$ is easy to estimate. For instance, we can use Lemma 3.2 and Corollary 3.4 in Giné and Zinn (1983) to show that this expectation is bounded from above and from below by fixed linear combinations of the following three quantities:

$$
\begin{gathered}
\delta_{0}:=\inf \left[t>0: n P\left\{I_{\left[X_{1} \in I_{j}\right]}>t\right\} \leq 1 / 72\right], \\
n \int_{\delta_{0}}^{\infty} P\left\{I_{\left[X_{1} \in I_{j}\right]}>t\right\} d t
\end{gathered}
$$

$$
\left(E\left|\sum_{i=1}^{n} \varepsilon_{i} I_{\left[X_{i} \in I_{j}\right]} I_{\left[I_{\left[X_{i} \in I_{j}\right]} \leq \delta_{0}\right]}\right|^{2}\right)^{1 / 2}
$$


which are respectively equal to

$$
I_{\left[p_{j}>1 / 72 n\right]}, \quad n p_{j} I_{\left[p_{j} \leq 1 / 72 n\right]} \text { and }\left(n p_{j}\right)^{1 / 2} I_{\left[p_{j}>1 / 72 n\right]} .
$$

Hence, from (7.6), (7.7) and (7.8) we conclude that conditions (7.2) and (7.3) are necessary and sufficient for

$$
n^{1-1 / p} E\left\|\sum_{i=1}^{n} \varepsilon_{i} f\left(X_{i}\right)\right\|_{\mathrm{BL}^{*}} \rightarrow 0
$$

By boundedness, $L_{1}$ convergence is equivalent to convergence in probability for this sequence, and (as mentioned above), equivalent to convergence in probability of the sequence $\left\{n^{1-1 / p}\left\|P_{n}-P\right\|_{\mathrm{BL} *}\right\}_{n=1}^{\infty}$. By de Acosta (1981, Theorem 3.1) (applied in the Banach space $\mathbf{L}_{1}$ ) this sequence converges a.s. if and only if it converges in probability.

7.2. REMARK. (1) The same proof shows that uniform boundedness of the quantities in (7.1) and (7.2) is equivalent to stochastic boundedness of the sequence (7.1). In particular this holds if $p_{j}=1 /|j|^{p}$.

(2) If $\sum p_{j}^{1 / p}<\infty$ then (7.2) and (7.3) hold; they also hold if $p_{j}=1 /|j|^{p} M(|j|)$ with $M \uparrow \infty$ and is slowly varying; but $\left\{p_{j}=1 /|j|^{p}\right\}$ does not satisfy (7.2) and (7.3).

(3) Consider

$$
\operatorname{Lip}_{1} \mathbf{R}=\left\{f: \mathbf{R} \rightarrow \mathbf{R}, \sup _{x \neq y}|f(x)-f(y)| /|x-y| \leq 1\right\} .
$$

For $\operatorname{Lip}_{1} \mathbf{R}$ we also have $\operatorname{Lip}_{1} \mathbf{R} \in \operatorname{CLT}\left(P, n^{1 / p}\right)$ if and only if $n^{1-1 / p}\left\|P_{n}-P\right\|_{\text {Lip, }} \mathbf{R}$ $\rightarrow 0$ in probability, and it is easy to show that this holds if and only if $t^{p} P\{|x|>t\} \rightarrow 0$ as $t \rightarrow \infty$. The proof is omitted.

(b) Weighted empiricals. Let $P$ be Lebesgue measure on $[0,1]$ and let $w(t), t \in$ $[0,1]$, be a $U$-shaped (weight) function on $(0,1)$, that is, a nonnegative measurable function that is nonincreasing on $(0, \gamma)$ and nondecreasing on $(\gamma, 1)$ for some $0<$ $\gamma \leq 1 / 2$. Let $\mathscr{F}=\left\{w(t) I_{[0, t]}: t \in(0,1 / 2)\right\} \quad$ by symmetry, it is enough to consider just half the $U$-shape. Assume moreover $\gamma=1 / 2$. The Cibisov-O'Reilly theorem states that $\mathscr{F} \in \operatorname{CLT}\left(P, n^{1 / 2}\right)$ if and only if $\mathscr{F}$ is $P$-pre-Gaussian, which amounts to a certain integral condition for $w$. In particular if $w(t)=o\left((t \ln \ln 1 / t)^{-1 / 2}\right)$ at zero then, $\mathscr{F} \in \operatorname{CLT}\left(P, n^{1 / 2}\right)$, but if $w(t)=O\left((t \ln \ln 1 / t)^{-1 / 2}\right)$ then $\mathscr{F}$ does not satisfy the CLT. The question is whether for these steeper weights convergence can be achieved if one divides by larger normings, say between $n^{1 / 2}$ and $n$. $w(t)$ is bounded for every $t$, so for normings $B(n)$ such that $B(n) / n^{1 / 2} \rightarrow \infty$ as $n \rightarrow \infty$, the finite dimensional distributions of $\left\{\sum_{i=1}^{n} \varepsilon_{i} w(t) I_{[0, t]}\left(U_{i}\right) / B(n)\right\}$, where $U_{i}$ are i.i.d. uniform random variables, tends to zero in probability. So, as for $\mathrm{BL}^{*}$, if $\mathscr{F} \in \operatorname{CLT}(P, B(n))$ the limit is necessarily 0 . We will only consider $B(n)=n^{1 / p}$, $p \in[1,2)$. As in the case of $\mathrm{BL}^{*}$, it is possible to obtain results from Proposition 5.7 about what weights $w$ give that the sequence

$$
\sup _{t \leq 1 / 2}\left|\sum_{i=1}^{n} w(t)\left(I_{[0, t]}\left(U_{i}\right)-t\right) / n^{1 / p}\right|
$$


converges to zero a.s. But Proposition 5.7 does not imply the complete answer, which is easy to obtain e.g. either by direct computation using order statistics and the law of iterated logarithm, or invoking a theorem of Pisier (1984) on VapnikCervonenkis classes. Pisier's result is as follows: let $\mathscr{C}$ be a class of measurable subsets of $(S, \mathscr{S})$, let $\left(\mathscr{M},\|\cdot\|_{\mathrm{TV}}\right)$ be the set of measures on $(S, \mathscr{S})$ of bounded total variation, with the total variation norm, and let $\|Q\|_{\mathscr{C}}=\sup _{C \in \mathscr{C}}|Q(C)|$, for $Q \in \mathscr{M}$. Then the identity map

$$
\left(\mathscr{M},\|\cdot\|_{\mathrm{TV}}\right) \rightarrow\left(\mathscr{M},\|\|_{\mathscr{C}}\right)
$$

is of type 2 if and only if $\mathscr{C}$ is Vapnik-Červonenkis.

7.3. THEOREM. Let $w(t), t \in(0,1 / 2)$, be a nonnegative nondecreasing function and let $\left\{U_{i}\right\}_{i=1}^{\infty}$ be independent random variables uniformly distributed on $[0,1]$. Then, for $p \in[1,2)$,

(1) The sequence (7.9) is stochastically bounded if and only if $\sup _{t \in(0,1 / 2]} t^{1 / p} w(t)$ $<\infty$

(2) The sequence (7.9) converges to zero in probability if and only if

$$
\lim _{t \rightarrow 0} t^{1 / p} w(t)=0 \text {. }
$$

(3) The sequence (7.9) converges to zero a.s. if and only if

$$
\int_{0}^{1 / 2}(w(t))^{p} d t<\infty .
$$

PROOF. As usual, we can randomize and consider

$$
\left\{\sum_{i=1}^{n} \varepsilon_{i} w(t) I_{[0, t]}\left(U_{i}\right)\right\}_{i=1}^{\infty}
$$

instead of $\left\{\sum_{i=1}^{n} w(t)\left(I_{[0, t]}\left(U_{i}\right)-t\right)\right\}_{n=1}^{\infty}$ since the necessary tail condition for $w(t)\left(I_{[0, t]}(U)-t\right)$ already implies boundedness of $\sup _{t>0} t^{1 / p} w(t)$. Consider the processes $X_{i}(t)=\varepsilon_{i} w(t) I_{[0, t]}\left(U_{i}\right), 0<t \leq \frac{1}{2}, X_{i}(t)=0$ for $t=0$ and for $t \in\left(\frac{1}{2}, 1\right]$, $i=1, \ldots X_{i}(t)$ has all of its trajectories of bounded variation, and the total variation norm of $X_{i}(t)$ is $2 w\left(U_{i}\right)$. So, these random processes define i.i.d. random measures $\nu_{i}(\omega)$ of bounded variation on $[0,1]$ that have total variation norm $2 w\left(U_{i}\right)$. The map (7.10) with $(S, \mathscr{S})=([0,1], \mathscr{B})$ and $\mathscr{C}=\{[0, t]: t \in[0,1]\}$ is of type 2. It is routine to check then that the type 2 inequality allows for the use of the classical computations for the CLT in the line, the stable case, about dominating truncated second moments by tails, to obtain:

$\sup _{t>0} t^{p} P\left\{\left\|X_{1}\right\|_{\mathrm{TV}}>t\right\}<\infty \Rightarrow$ the sequence (7.11) is stochastically bounded and

$t^{p} P\left\{\left\|X_{1}\right\|_{\mathrm{TV}}>t\right\} \rightarrow 0 \Rightarrow$ the sequence (7.11) converge to zero in pr.

But $t^{p} P\left\{\left\|X_{1}\right\|_{\mathrm{TV}}>t\right\}=t^{p} P\left\{2 w\left(U_{1}\right)>t\right\}=t^{p} w^{-1}(t / 2)$ where $w^{-1}(s)=$ $\sup \{t: w(t) \geq s\}$. Thus the direct implications in (1) and (2) are proved. For the converse in (1) and (2), note that, using Lévy's inequality,

$$
\begin{aligned}
& P\left\{\left\|\sum_{i=1}^{n} X_{i}(t)\right\|_{\infty}>c n^{1 / p}\right\} \geq P\left\{\max _{i \leq n}\left\|X_{1}(t)\right\|_{\infty}>c n^{1 / p}\right\} \\
& =P\left\{\max _{i \leq n} w\left(U_{i}\right) \geq c n^{1 / p}\right\} \geq n w^{-1}\left(c n^{1 / p}\right) /\left(1+n w^{-1}\left(c n^{1 / p}\right)\right) .
\end{aligned}
$$


Finally, (3) follows from (2) by de Acosta (1981, Theorem 3.1).

\section{REFERENCES}

1. A. de Acosta (1981), Inequalities for B-valued random vectors with applications to strong laws of large numbers, Ann. Probab. 9, 157-161.

2. A. de Acosta, A. Araujo and E. Giné (1978), On Poisson measures, Gaussian measures and the central limit theorem in Banach spaes, Advances in Probability and Related Topics, Vol. IV (J. Kuelbs, ed.), Dekker, New York, pp. 1-68.

3. N. T. Andersen and V. Dobric (1987), The central limit theorem for stochastic processes, Ann. Probab. 15, 164-177.

4. N. T. Andersen, E. Gine, M. Ossiander and J. Zinn (1988), The central limit theorem and the law of iterated logarithm for empirical processes under local conditions, Probability Theory and Related Fields, 77, 271-308.

5. G. W. Bennett (1962), Probability inequalities for sums of bounded random variables, J. Amer. Statist. Assoc. 57, 33-45.

6. M. Csörgö, S. Csörgö, L. Horvath and D. M. Mason (1986), Normal and stable convergence of integral functions of the empirical distribution function, Ann. Probab. 14, 86-118.

7. W. Feller (1971), An introduction to probability theory and its applications, Vol. II, 2nd ed., Wiley, New York.

8. E. Giné and J. Zinn (1983), Central limit theorems and weak laws of large numbers in certain Banach spaces, Z. Wahrsch. Verw. Gebiete 62, 323-354.

9. E. Giné and J. Zinn (1986), Empirical processes indexed by Lipschitz functions, Ann. Probab. 14, 1329-1338.

10. E. Giné and J. Zinn (1984), Some limit theorems for empirical processes, Ann. Probab. 12, 929-989.

11. E. Gine and J. Zinn (1986), Lectures on the central limit theorem for empirical processes, Lecture Notes in Math., vol. 1221, Springer-Verlag, New York and Berlin, pp. 50-113.

12. B. Heinkel (1983), Majorizing measures and limit theorems for $c_{0}$-valued random variables, Lecture Notes in Math., vol. 990, Springer-Verlag, New York and Berlin, pp. 136-149.

13.,$(1987)$, Some exponential inequalities with applications to the central limit theorem in $C[0,1]$ (to appear).

14. J. Høffmann-Jorgensen (1984), Stochastic processes on Polish spaces (to appear).

15. N. Jain and M. Marcus (1975), Central limit theorem for $C(S)$-valued random variables, J. Funct. Anal. 19, 216-231.

16. D. Juknevicienè (1986), On the central limit theorem in the space $c(s)$ and majorizing measures, Lietuvos Mat. Rink. 26, 362-373.

17. J. Kuelbs and J. Zinn (1979), Some stability results for vector valued random variables, Ann. Probab. 7, 75-84.

18. M. Ledoux (1982) Loi du logarithme itéré dans $C(S)$ et fonction characteristique empirique, $\mathrm{Z}$. Wahrsch. Verw. Gebiete 60, 425-435.

19. M. Ledoux and M. Talagrand (1986), Characterization of the law of the iterated logarithm in Banach spaces, Ann. Probab. (to appear).

20. V. Mandrekar and J. Zinn (1980), Central limit problem for symmetric case: Convergence to non-Gaussian laws, Studia Math. 67, 279-296.

21. M. B. Marcus (1987) $\xi$-radial processes and random Fourier series, Mem. Amer. Math. Soc., vol. 68 , no. 368 .

22. M. Marcus and G. Pisier (1984), Characterization of almost surely continuous p-stable random Fourier series and strongly stationary processes, Acta Math. 152, 245-301.

23. M. Marcus and G. Pisier (1984), Some results on the continuity of stable processes and the domain of attraction of continuous stable processes, Ann. Inst. H. Poincaré 20, 177-199.

24. V. Panlauskas and A. Raxkauskas (1984), On operators of state type, Lietuvos Mat. Rink. 24, 145-159.

25. G. Pisier (1984), Remarques sur les classes de Vapnik-Červonenkis, Ann. Inst. H. Poincaré Probab. Statist. 20, 287-298.

26. W. Stout (1974), Almost sure convergence, Academic Press, New York. 
27. M. Talagrand (1987), Regularity of Gaussian processes, Acta Math. 159, 99-149.

28. __ (1986), Necessary conditions for sample boundedness of $p$-stable processes (to appear).

Aarhus Universitet, Matematisk Institut, DK-8000 Aarhus C, Denmark Texas a\&M University, Department of Mathematics, College Station, TEXAS 77843 